\title{
Development of an apparatus to quantify the volitional muscle performance of rat plantar flexors in vivo
}

Vineet Shastri

West Virginia University

Follow this and additional works at: https://researchrepository.wvu.edu/etd

\section{Recommended Citation}

Shastri, Vineet, "Development of an apparatus to quantify the volitional muscle performance of rat plantar flexors in vivo" (2001). Graduate Theses, Dissertations, and Problem Reports. 1135.

https://researchrepository.wvu.edu/etd/1135

This Thesis is protected by copyright and/or related rights. It has been brought to you by the The Research Repository @ WVU with permission from the rights-holder(s). You are free to use this Thesis in any way that is permitted by the copyright and related rights legislation that applies to your use. For other uses you must obtain permission from the rights-holder(s) directly, unless additional rights are indicated by a Creative Commons license in the record and/ or on the work itself. This Thesis has been accepted for inclusion in WVU Graduate Theses, Dissertations, and Problem Reports collection by an authorized administrator of The Research Repository @ WVU. For more information, please contact researchrepository@mail.wvu.edu. 
Development of an Apparatus to Quantify the Volitional Muscle Performance Of Rat Plantar Flexors In Vivo

\author{
Vineet Shastri, B.S. \\ Thesis submitted to \\ College of Engineering and Mineral Resources \\ West Virginia University
}

In Partial Fulfillment of the Requirements for the

Degree of Master of Science

In Industrial Engineering

Rashpal S. Ahluwalia, Ph.D., Chair

Greg Cutlip, Ph.D.

Dianne McMullin, Ph.D.

Industrial and Management Systems Engineering Department

Morgantown, WV, 2001

Keywords: Biomechanics, Rodent Dynamometer, Muscle Performance, Plantar Flexors 


\section{ABSTRACT \\ Development of an Apparatus to Quantify the Volitional Muscle Performance of Rat Plantar Flexors In Vivo Vineet Shastri}

An in vivo animal model was developed to study the effects of voluntary eccentric, concentric, and isometric muscle actions and varying work-rest cycles on muscle performance, behavior, and histological and biochemical response. Using a custom-designed apparatus that was attached to a standard operant chamber, rats were operantly conditioned with food rewards to perform a voluntary lifting task to generate controlled movement of the plantar flexors. An opening in the front panel of the operant chamber allowed the rat to enter a Plexiglas tube that was mounted vertically to restrict the movement of the rat. A load cell was embedded in a platform at the bottom of the tube to measure the dynamic force exerted by the plantar flexors. Inside the tube, a neck ring was supported by a yoke that moved along two vertical shafts via linear bearings. A displacement transducer (LVDT) was attached to the weight pan to measure the range of motion of the lift, and allowed determination of velocity and acceleration of the lifting motion. The apparatus allowed the rat to enter the tube through the opening, insert its neck into the ring, and lift the ring assembly. The entire process was computer automated, and vertical displacement, time during each lift, and dynamic forces exerted during each lift were sampled at $100 \mathrm{~Hz}$ via a computer-controlled data acquisition system. This apparatus allows skeletal muscle performance to be studied longitudinally and in a controlled biomechanical environment. The apparatus is well suited to study the effect of chronic voluntary muscle actions and work-rest cycles on behavior and physiological outcomes. 


\section{ACKNOWLEDGEMENTS}

I take this opportunity to thank and show my appreciation to everyone who has had a role to play during my graduate career at West Virginia University. I would like to thank my research advisor Dr. Ahluwalia also the chair of the committee, without whose encouragement and guidance this thesis would not have been possible. To Dr. Cutlip, who accepted me on this project and advised me throughout my research work. Also, for his patience and the time he spent in developing my bioengineering skills and honing my engineering skills. To Dr. McMullin for serving on my committee and the advice and encouragement she provided.

To the members of the Musculoskeletal Pathomechanics Research Team at NIOSH for their support. Specially, to Dr. Wirth who helped me whenever I approached him without any hesitation. To Israel Hall for his help with the description of the electrical hardware. To Erik Gregory for his support and help with the design of the apparatus.

To all my friends for being so accommodating during my graduate studies. Finally, I would like to thank my parents S. R. Shastri and Geeta Shastri and my sister Tina for their love and encouragement and the financial support that they provided during my graduate studies. 


\section{Table of Contents}

Title Page

Abstract

i

Acknowledgements

ii

Table of Contents

iii

List of Tables

iv

List of Figures

vi

vii

1. Literature Review 1

1.1 Introduction 1

1.2 Muscle Mechanics 2

1.3 Experimental Models 3

1.3.1 In Vitro Models 3

1.3.2 In Situ Models

1.3.3 In Vivo Models 6

1.4 Problem Description 8

1.5 Scope of Research $\quad 8$

2. System Description 9

2.1 Introduction 9

2.2 Dynamometer Description 9

$\begin{array}{ll}\text { 2.2.1 System Flowchart } & 10\end{array}$

2.2.1.1 Overall Setup 11

2.2.2 System Overview 13

2.2.2.1 Base 13

2.2.2.2 Force Plate 15

2.2.2.3 Plexiglas Tube 16

2.2.2.4 Pans 18

2.2.2.5 Neck Ring 20

2.2.2.6 Support 21

2.2.2.7 Stoppers $\quad 22$

2.3 Hardware 22

2.3.1 Load Cell 22 
2.3.2 Load Cell Amplifier $\quad 23$

2.4 Transducer Selection 23

2.4.1 Ankle Mechanics 23

2.4.2 Displacement Transducer 24

2.4.2.1 Transducer Sleeve 25

2.4.3 Signal Conditioner 25

2.4.4 Teflon Support $\quad 25$

$\begin{array}{ll}2.5 \text { Software } & 26\end{array}$

2.6 Computer System Interface 28

2.6.1 PCI- MIO- 16XE- 10 A/D Board 29

2.7 Accessory Devices $\quad 30$

2.7.1 Pellet Dispenser $\quad 30$

2.7.1.1 L- Frame Support $\quad 30$

2.7.2 Fan $\quad 30$

2.7.3 White Noise Emitter $\quad 30$

2.7.4 Light Emitting Diode 31

2.7.5 Head Entry Infrared Switch 32

2.7.6 Nose Poke Infrared Switch 31

2.7.7 House Light 31

2.8 Triple Output Power Supply 32

2.9 Nose Poke Fixture 33

3. Calibration and Testing 34

3.1 Transducer and Signal Conditioner 35

3.2 Load Cell 36

3.3 Operant Conditioning 37

3.3.1 Pseudocode for Operant Conditioning 37

3.4 Testing 36

3.4.1 Pseudocode for Testing 39

3.5 Flowchart for Dynamic Mode 42

3.6 Flowchart for Static Mode $\quad 44$

4. Conclusion 43

4.1 Conclusion 46 
4.2 Recommendations 46

$\begin{array}{ll}\text { References } & 48\end{array}$

Appendix A - LabVIEW Code

$\begin{array}{ll}\text { Virtual interface } & 51\end{array}$

Code for the Static Mode $\quad 52$

Code for the Dynamic Mode $\quad 54$

\section{LIST OF TABLES}

Table $1 \quad$ LVDT Calibration Data 35

Table 2 Load Cell Calibration Data 36 


\section{LIST OF FIGURES}

Figure1. Isometric View of the Dynamometer 10

Figure 2. Schematic View of the Setup 11

$\begin{array}{ll}\text { Figure 3. Overall setup } & 12\end{array}$

Figure 4. Set up with the transducer $\quad 12$

Figure 5. Front and top views of base 14

Figure 6. Front and top views of the force plate 15

$\begin{array}{ll}\text { Figure 7. Front and profile views of the Plexiglas tube } & 17\end{array}$

$\begin{array}{ll}\text { Figure 8. Isometric view of the dynamometer } & 18\end{array}$

Figure 9. Top view of weight pan 19

Figure 10. Front view of dynamometer 20

Figure 11. Drawing of the dynamometer 21

Figure 12. Support for shafts, stimulus light, and nose poke device 22

Figure 13. Schematic view of the ankle rotation 24

Figure 14. Front panel or graphic user interface 26

Figure 15. Wiring diagram 28

Figure 16. Top, profile and front views of the L - frame 31

Figure 17. Modified nose poke device with the support 34

Figure 18. LVDT calibration curve 36

Figure 19. Load cell calibration curve $\quad 37$

Figure 20. Flowchart for the dynamic process 42

Figure 21. Flowchart for the static process $\quad 44$ 


\section{CHAPTER 1 INTRODUCTION}

\subsection{Introduction}

Musculoskeletal disorders due to overexertion and repetitive motion continue to be the leading cause of lost work time and compensation claims [7,18,21,22,30]. Estimates of costs range from $\$ 13$ to \$20 billion annually [42]. Recently a study reported there were approximately 705,800 cases of overexertion and repetitive motion injury in the United States that led to days away from work [42]. Many researchers have related occupational injury incidence to job demands, however, there is a lack of experimental evidence that directly links loads and the conditions under which they are lifted to the incidence of musculoskeletal injuries [26]. Many researchers have established databases of maximal and acceptable criterion, but the evidence concerning safe practice is still conflicting. Lifting guidelines tend to be based on problems of litigation rather than physiological, biomechanical or psychophysical findings.

The four primary methods addressing lifting capacity are: 1) the biomechanical approach, 2) the psychophysical approach, 3) the epidemiological approach, and 4) the physiological approach [16]. These methods use different criteria to establish the safety limits for lifting tasks. Stresses imposed on the musculoskeletal system from external and internal loads and their correlation to injury serves as the criteria in the biomechanical approach [10]. The physiological approach relates the energy expenditure to injury incidence and the basis for the lifting capacity in the psychophysical approach is that the subject adjusts the load so that overexertion does not occur [5]. The epidemiological approach identifies factors that are positively or negatively associated with the development or recurrence of adverse medical conditions to recommend appropriate work practices to mitigate injury incidence [26].

However, the current approaches do not incorporate soft tissue injury mechanisms into their models. Muscle injury has been well studied in human and animal models within the past 15 to 20 years. The understanding of causal factors associated with muscle injury and the resultant histopathological process would be beneficial to increase the robustness of current models such that injury incidence can be further ameliorated.

\subsection{Muscle Mechanics}

Damage to active muscles is a common occurrence at work places and in sports. Skeletal muscle performs multiple functions, and the often-discussed function in physiology is the mechanical function [34]. To accomplish movements, skeletal muscles have to function as motors for muscle shortening, stabilizers for tension development and shock absorbers to absorb energy and prevent injuries. The motor and the stabilizer functions relate to the concentric and isometric actions of muscles [34]. 
As a muscle exerts force, it generates a moment (torque), on the involved joint, along the line of application of the muscle force. The moment is calculated as the product of the muscle force and the perpendicular distance between its point of application and the center of motion [32]. This distance is known as the lever arm or moment arm. Muscle contractions can be classified according to the relationship between either the muscle tension and the resistance to be overcome or the muscle moment generated and the resistance to be overcome.

The two types of muscle contraction are isotonic and isometric. In an isotonic contraction, muscle tension remains constant as the muscle changes length. In an isometric contraction, the muscle is prevented from shortening, so the development of tension occurs at constant muscle length. There are two types of isotonic contraction- concentric and eccentric. In both cases, the muscle changes length at constant tension. However, when a muscle develops sufficient tension to overcome the load on the muscle, the muscle shortens and causes joint movement. This contraction is called a concentric contraction [32]. The net moment generated by the muscle is in the same direction as the change in joint angle. When a muscle cannot develop sufficient force and is overcome by the external load, it progressively lengthens instead of shortening, in which case the muscle is said to contract eccentrically [32]. The net muscle moment is in the opposite direction from the change in joint angle.

Injury criteria should relate to muscle function. Muscle function is the ability to exert force under a given set of conditions (range of motion, given velocity and a given level of activation). "Muscle injury may be defined as a prolonged impairment of the ability of a muscle to produce force"[36]. The probability of a muscle being injured is highest during the eccentric contraction of the muscle and it can cause histopathological changes in the muscle fibers [2,20,25]. Muscle injury due to eccentric contractions involves high mechanical forces of stretch [34].

Thus, mechanical damage usually occurs when muscles absorb energy. Eccentric contractions are known to cause greater damage in muscles suggesting that high load tensions in fibers may be more important than physiologic considerations in the etiology of the injury process [3]. Muscle damage is accompanied by a loss of contractile force with visible damage to the muscle fibers.

Over the past 20 years, several soft tissue injury models (both human and animal) have been developed, to elucidate the dynamic parameters (number of repetitions, range of motion, velocity, acceleration, type of muscle action and muscle force) responsible for skeletal muscle injury. The soft tissue injury models also have addressed the sites of injury, time course of inflammation and repair, performance decrement due to injury and time course of performance reestablishment, and histological and biomechanical expressions due to high force muscle contractions. An in-depth understanding of soft tissue pathomechanics may assist in further refinement of the current ergonomic lifting models. One goal is to design jobs so that loading of the muscle is within a specified safety limit. 


\subsection{Experimental Models}

Researchers over the years have tried to induce injury to muscles in an effort to study injury mechanisms and properties of muscles. Conventional techniques used to study the contractile properties of skeletal muscles have been highly invasive [36]. The techniques required the muscle to be exposed and the experiments were conducted either in vitro or in situ. Another method commonly used to study muscles is the in vivo preparation. In in vitro preparations, the muscle is completely isolated from the animal and tested in a physiologic bath. In in situ preparations, the muscle remains within the body and retains normal vascular and neural supply but the nerve and tendon are exposed [36]. In contrast, in in vivo preparations, the muscle-tendon complex in addition to the neural and vascular supply is intact. Thus muscles can be tested in a non-invasive manner. Injury is induced without surgery in the in vivo technique. One advantage of the in situ and in vitro preparations over the in vivo preparation is that the muscle properties of a specific muscle can be determined [36].

\subsubsection{In vitro Models}

In the in vitro preparation, the muscle is excised from the body and immersed in a physiologic solution. The muscle of interest is then attached to a force transducer to make the necessary measurements. It is true that the most intensive studies of muscle contractile properties have been carried out using the in vitro preparations. These studies have contributed to a better understanding of the muscle as a functional unit.

Investigations of muscle usually include the force-frequency experiments to characterize the muscle output force as a function of stimulation frequency. The in vitro preparation lends itself well for conducting the force-frequency experiments. One of the essential components of in vitro preparations is the stimulating electrode chronically implanted into the muscle. This enables control of the activation pattern of the target muscle. Usually Teflon-coated platinum electrodes are implanted in the muscle under study and stimulated at certain frequencies for different lengths of time. Since muscles are isolated from the body in the in vitro method, force generated by the isolated muscles can be determined. The isometric force-length curve defines the steady state property of a muscle tissue and this property can be best studied when activation and muscle fiber length are held constant [38]. The in vitro method can be used to control the length of stretch, velocity, and force and the activation pattern.

Muscle contractions studied in vitro are involuntary and for the maximum force to be obtained, the stimulation parameters have to be optimized. The stimulation parameters include voltage or current, frequency and pulse duration. In addition, the optimal value of frequency needs to be determined before and after an injury has been induced [36]. According to a study by Warren et al. [36], the viability of the 
in vitro preparation has to be considered because muscles weighing more than $30 \mathrm{mg}$ cannot be studied in vitro because sufficient quantities of nutrients like oxygen may not be able to diffuse to the core of the muscle held at physiological temperatures in baths. Also, muscles studied in vitro should be maintained at optimal length because proteins in the muscles degrade differently at different lengths.

In a study by Lowe et al. [24], anterior crural muscles of a rat were injured in vivo and then isolated and studied for various factors including the protein metabolism. The protein synthesis rates were observed to increase 48 hours after injury was induced. Maintaining the muscle in a bath for long periods of time raises doubts about the viability of the in vitro preparation.

In yet another study by Warren et al. [35] concerning the muscle to bone relationship, the Extensor Digitorum Longus (EDL) and the tibia were exercised in vivo and later isolated. But the EDL was the only muscle that could be studied in vitro because excising the tibialis anterior muscle without damaging the tibia is not possible [35] and also because of the small size of the extensor hallucis longus muscle. Hence it is extremely difficult to isolate muscles without damaging the functional structure of the body.

Since muscles in the in vitro preparations are isolated and held in physiological baths, they are well suited to understand the functioning of the muscle alone, though from a physiological standpoint the study of the synergistic properties of the muscle is compromised. Therefore, this method does not permit the study of muscle-tendon interactions. In vitro method is an acute exposure method and does not permit chronic studies.

In addition, when muscle fibers are ruptured or membranous components are disrupted, proteins and enzymes are released into the vascular compartments. The muscle relative to the tendons, nerve tissues, vascular vessels and the motor units cannot be investigated because the muscle is isolated from the body.

\subsubsection{In situ Models}

Another form of invasive method used to study muscle mechanics and contraction induced injury is the in situ preparation of the muscle [11]. Some of the drawbacks faced with the in vitro preparation are overcome by this method. The in situ preparation involves keeping the muscle within the body with the vascular supplies intact. An incision is made to insert stimulation electrodes and experiments are carried out allowing the muscle and other systems to perform as one unit. Evidence of muscle damage includes disruption of muscle fibers [13,17,27] increased levels of muscle proteins [8,14,28,29] and changes in muscular force output. Keeping the muscle intact facilitates the study of protein level change, change in output force and the varying patterns of the motor unit recruitment. When the muscle is intact and injuries are induced through stimulation, the exact nature of the injury independent of the task or 
exercise performed can be observed in detail. The specific conditions of injury can also be studied through control of the mechanical insults.

According to a study by Faulkner et al. [12], the muscle membrane appears to be damaged to a greater extent in in vitro experiments than in in situ experiments. The in situ method overcomes the need to isolate the muscle reducing the risk of damage to the muscle. Faulkner and Jones [11] developed an apparatus for in situ exercise of the lower limb of small mammals to study the effects of different types of contraction on the skeletal muscle. Contractions were performed using a roller pump as the drive mechanism and setting the speed control on the pump controlled velocity.

In a study of the dorsiflexor muscles of a rabbit by Patel et al. [31], electrical stimulation was delivered to the target muscle when the animal was in an anesthetized state. The tendons were isolated and attached to motors. This could have caused soreness in the muscle. Muscle soreness is used as one of the biomarkers for muscle injury. In circumstances where invasive techniques such as incisions have been made or tendons ligated or electrical stimulators inserted could affect the inflammation response thus leading to erroneous results.

Gregor et al. [15] conducted a study in which the individual muscle force, length and velocity were analyzed during gait. Data for an average step cycle at certain speeds were compared to the forcevelocity properties obtained from the same muscle under stimulation in situ. The results indicated that the force and power generated in the in vivo preparation were higher than the force and power generated in the in situ preparation. The reason cited for the differences is that it might be due to the recovery of strain energy.

Since in situ preparations involve partially intact muscles and the electrical stimulation does not recruit the entire muscle, incongruous areas at the injury site could compromise the validity of this type of investigation. Since force is developed about a joint and the in situ preparation involves isolating the tendon the force developed may not be the actual force that would have been utilized by the body to overcome the load. Muscles and the tendons function as one entity [36]. Moreover, the musculotendon unit has the ability to store and release elastic energy. Thus, this energy cannot be measured when the tendon is separated and this energy gets dissipated as heat.

Thus using a more intact preparation to characterize the muscle-tendon synergist response, Willems et al. [37], used a custom built dynamometer to determine the performance of electrically stimulated plantar flexor muscles working in situ in the intact rat. The experiment involved inserting an electrode along the tibial nerve of an anaesthetized rat. This type of an experiment that does not involve isolating the muscle allows the study of the muscles and tendons as a functional unit. The proteins and enzymes that spill into the vascular vessels can be used as biomarkers of injury. Also the synergistic 
properties of the muscle can be investigated. But the rat being anaesthetized, the in situ exercise becomes an involuntary act.

\subsubsection{In vivo Models}

The in vivo preparation is an improvement over the in vitro and in situ procedures because it uses noninvasive techniques and allows the study of muscle-tendon synergists and the study of chronic muscle actions. The muscle-tendon complex is allowed to remain inside the body with its vascular supplies and the nervous system intact and then injury is induced in vivo. In in vivo preparations, torque is measured across a joint. Hence the synergistic properties of the muscle can be distinctly observed [36].

Various training models using non-invasive techniques have been developed to induce injury to various muscles. These include treadmills [15], and dynamometers for humans and animals [1,4,6], wheel running for mice and weight placed on the wings of chickens [33]. Treadmill exercises and wheel running exercises are total body physical activities in which it is difficult to assess the damage to the muscle because of the diversity of actions being performed. It is clear that the force exerted by the muscles cannot be measured accurately using these techniques. Thus, there is a clear need to study muscle response due to controlled biomechanical exposures.

Servomotors have been used to investigate the ankle muscle reflexes in humans [4] but no methods are available to measure the voluntary muscular forces about a given joint in small mammals and to study the synergistic properties of the muscle. Ashton-Miller [4] built one such device to study the biomechanical behavior of the dorsi and plantar flexor muscles of the ankle in vivo. The rat's foot was strapped to an adjustable footplate, which was connected to a cylinder designed to serve as a torque cell. The cylinder measured the moment of the ankle muscle about the ankle joint and was connected to the shaft of a servomotor. When the footplate was rotated through a predetermined angle the torque cell recorded the resistance offered by the anesthetized rat. The isometric force was calculated by dividing the measured maximal ankle movement by the muscle lever arm. To validate the measurements, the force output in vivo was compared with the force values determined in situ. The values were found to be within $10 \%$ of each other.

Dynamometers have been used to evaluate the effects of training and recuperation on muscle performance. In a dynamometer developed by Cutlip et al. [9] to study the responses of the plantar flexor muscles of a rat and also to measure the forces developed at the plantar surface of the foot, the anaesthetized rat was placed on a table and its foot was held in a specially designed fixture. The muscle was stimulated using platinum electrodes and a stimulator. Although this model was sufficient to measure forces, the use of anaesthesia and its effects on the nervous system are questionable. The type of anaesthetic regimen, the concentration of anaesthesia and the duration anaesthesia has been administered 
all influence muscle force. Some of the more common anaesthetics have been known to have adverse effects on rats [36]. Though there is not much evidence to support the fact, anaesthetics might affect the release of proteins and enzymes when the muscle fibers are ruptured.

Later, models similar to the ones developed by Ashton-Miller [4] and Cutlip et al. [9] were extended to studies on other animals. In the in vivo noninvasive eccentric-contraction treatment used by Patel et al. [31], a rabbit was anaesthetized and electrical stimulators were implanted along the muscle. Each foot was secured to a footplate connected to a servomotor, which was activated to induce injury.

Warren et al. [35] studied the anterior crural muscle and tibial bone relationship in mice. In the first stage, an in vivo eccentric contraction was performed on the anterior crural muscle of the mouse. The apparatus used was a modified version of the model built by Ashton-Miller [4] described previously. Immediately after the in vivo protocol, the EDL muscle was isolated. According to Faulkner [12] et al., enzyme release occurs within an hour after injury when muscles are in vitro but is delayed several days when muscles are in vivo. According to Lieber [23] et al., the magnitude of loss of the cytoskeletal protein desmin in rabbits was approximately correlated with the loss of contractile force 1-3 days after injury. It would be futile to speculate the effects of injury by isolating the muscle immediately after in vivo exercises have been performed by the animal.

In another study of the mouse anterior crural muscles of mice by Lowe et al. [24], it was observed that protein synthesis rate increased 48 hours after injury. It is possible only through the use of noninvasive in vivo techniques [keeping the animal alive] to study the complete cycle of degeneration and regeneration of the muscle fibers after an injury is induced. The indirect measures of muscle damage used in studies include enzyme release from muscle fibers, a decrease in the maximum power and in human beings the report of muscle soreness [12].

Dynamometry has been widely applied in humans but rarely in animals [37]. A contraption was developed by Klitgaard [19] to study the effect of load on the muscles of the torso and hind limb. The rat would enter a Plexiglas tube mounted vertically on a platform and insert its neck into a ring that was connected to a rod hinged at one end and weights hung on the other end. The rat was trained to stand on its hind limbs and rise to its full height. On doing so, the rat was rewarded with a food pellet. Since this contraption was not designed to measure force, Klitgaard used the following technique to determine the force generated by the muscle. The force used by the rat for each lift was a function of the angular velocity and the angular acceleration of the rod. The angle of the force relative to the rod depends on the angle of deflection at any time in each lifting. These calculations used by Klitgaard to determine the force compromised the accuracy of the force value generated by the muscle. Also in estimating the force other factors had to be considered such as the lifting height. A force factor was introduced because the hinge joint locked the lever, when the rat stopped lifting. Klitgaard used a plotter attached at the end of 
the lever to determine the height. Although the rats could be tested for volitional movements, the study was limited to the concentric action of the muscle.

The lack of in vivo models that would address all the factors stated in the above study is a concern. Though most intensive studies have been conducted using in situ and in vitro preparations, which involve invasive procedures, there is a paucity of studies that have dealt with all the factors that have been discussed so far in this material. Hence there is a need to develop an apparatus that will measure the volitional muscle performance with the muscles intact and without surgery and also facilitate chronic studies of muscle.

\subsection{Problem Description}

The objective of this research was to develop an apparatus to quantify the volitional muscle performance of rat plantar flexor in vivo. A dynamometer was required that could measure and display force in real time. Using noninvasive techniques, the rat can be tested on a dynamometer that can measure time, displacement and force generated by the muscle. With a noninvasive in vivo assessment, multiple exposures are possible, the threshold of injury can be determined easily, and the time course of degeneration and regeneration of muscle fibers can be estimated.

\subsection{Scope of the Research}

After an extensive literature research, the design for a suitable apparatus was developed. The dynamometer was fabricated on the premises and the apparatus was setup using commercially available external devices like the test chamber and white noise, house light and stimulus light. The dynamometer was calibrated and tested. Data acquisition and control program using the LabVIEW software was developed and deployed to automate the data acquisition and control process. Necessary changes were made to the prototype hardware and software to obtain optimal performance. 


\section{CHAPTER 2 SYSTEM DESCRIPTION}

\subsection{Introduction}

An in vivo animal model was developed to study the effects of volitional eccentric, concentric, and isometric muscle actions and varying work-rest cycles on muscle performance, behavior, and histological and biochemical response. Using a custom-designed apparatus that was attached to a standard operant chamber, rats were operantly conditioned with food rewards to perform a voluntary lifting task to generate controlled movement of the plantar flexors. An opening in the front panel of the operant chamber allowed the rat to enter a Plexiglas tube that was mounted vertically to restrict the movement of the rat. A load cell was embedded in a platform at the bottom of the tube to measure the dynamic force exerted by the plantar flexors. Inside the tube, a neck ring was supported by a yoke that moved along two vertical shafts via linear bearings. A displacement transducer (LVDT) was attached to the weight pans to measure the range of motion of the lift, and allowed determinations of velocity and acceleration of the lifting motion. The apparatus allowed the rat to enter the tube through the opening, insert its neck into the ring, and lift the ring assembly. In this way, eccentric and concentric muscle contractions were produced. In some cases, weights were placed on pans attached to the ring assembly to vary the load. In other cases, movement of the yoke was locked at different heights such that multipositional isometric performance could be studied. The entire process was computer automated, and vertical displacement, time during each lift, and dynamic forces exerted during each lift were sampled at $100 \mathrm{~Hz}$ via a computer-controlled data acquisition system. This model allows skeletal muscle performance to be studied longitudinally and in a controlled biomechanical environment. This model also is well suited to study the effect of chronic volitional muscle actions and work-rest cycles on behavioral and physiological outcomes.

\subsection{Description of the Dynamometer}

The dynamometer is computer controlled and the data collection is automated, thus completely eliminating operator intervention. The dynamometer is designed to quantify the volitional static (isometric) and dynamic (concentric and eccentric) performances of the plantar flexors. The static design mode facilitates the measurement of tension at a constant muscle length. In the dynamic mode, the dynamometer measures the force in the concentric mode in which the muscle contracts developing sufficient tension to overcome the load on the muscle and the eccentric mode in which the muscle lengthens without being able to develop sufficient force to overcome the load. The data acquisition 
program is used to sample the load cell, displacement transducers, the nose poke device and the head entry device.

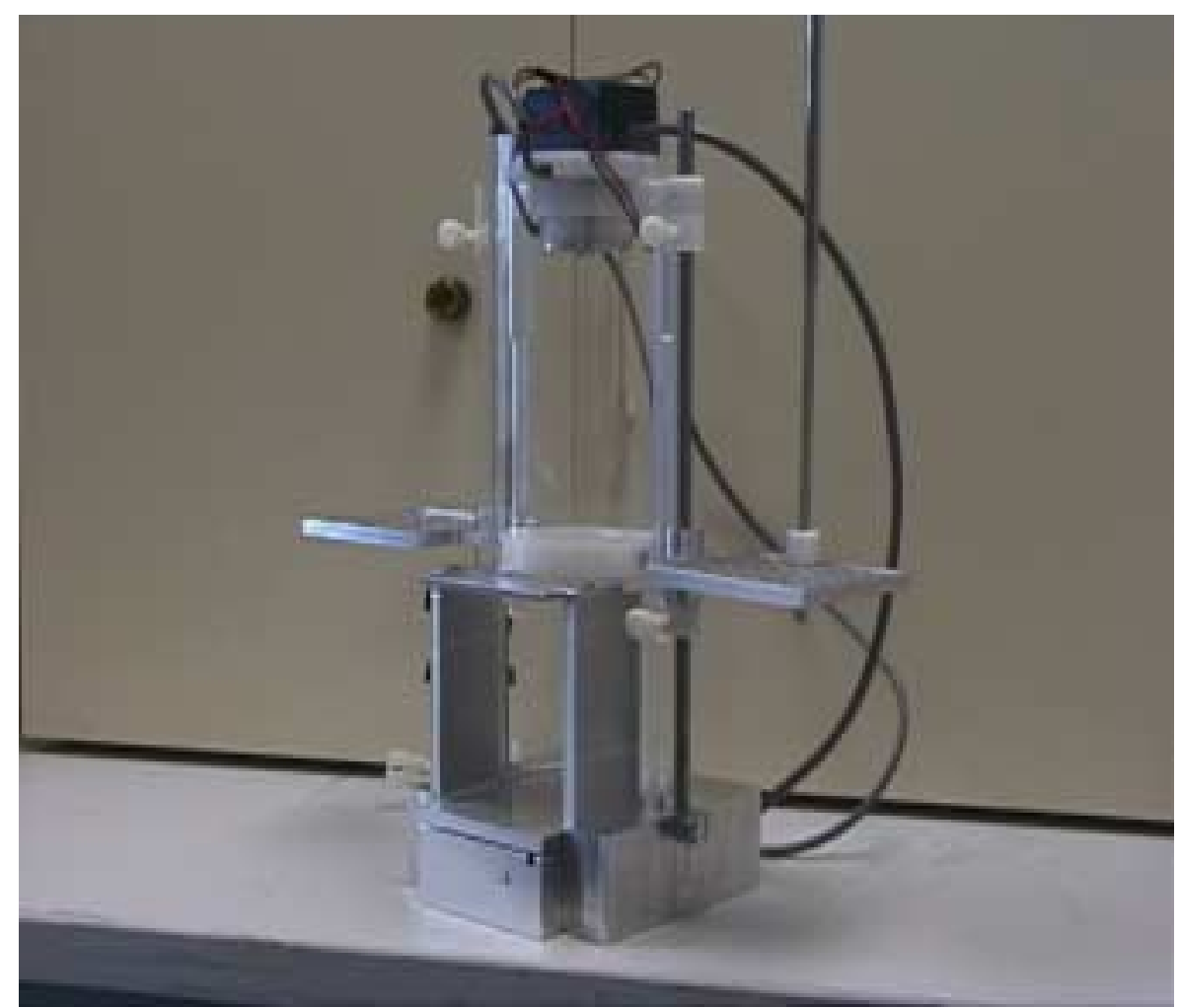

Figure 1- Isometric View of the Dynamometer

The output devices like the food pellet dispenser, fan, white noise, house light, and the stimulus light are also controlled using the computer. Weight pans have been constructed so that the rat can lift the weight placed in these pans on its shoulders and cause sub maximal tension in the plantar flexors to induce injury. The weight pans have been designed to carry a maximum weight of $2.2 \mathrm{lbs}$. The dynamometer is equipped with a displacement transducer fitted to the weight pans to measure the range of motion of the response. The selected transducer can measure a change in length of up to an inch.

\subsubsection{System Flowchart}

Figure 2 depicts the system flowchart. The figure shows the profile view of the dynamometer with the LVDT and load cell attached to the computer through an A/D card. The nose poke device and the head entry device are attached to the computer through a relay card. The relay card also connects the outputs like the house light, white noise, fan, pellet dispenser, and stimulus light to the computer. The 
data acquisition system (computer and LabVIEW) samples the load cell and the LVDT at a rate of 100 Hz.

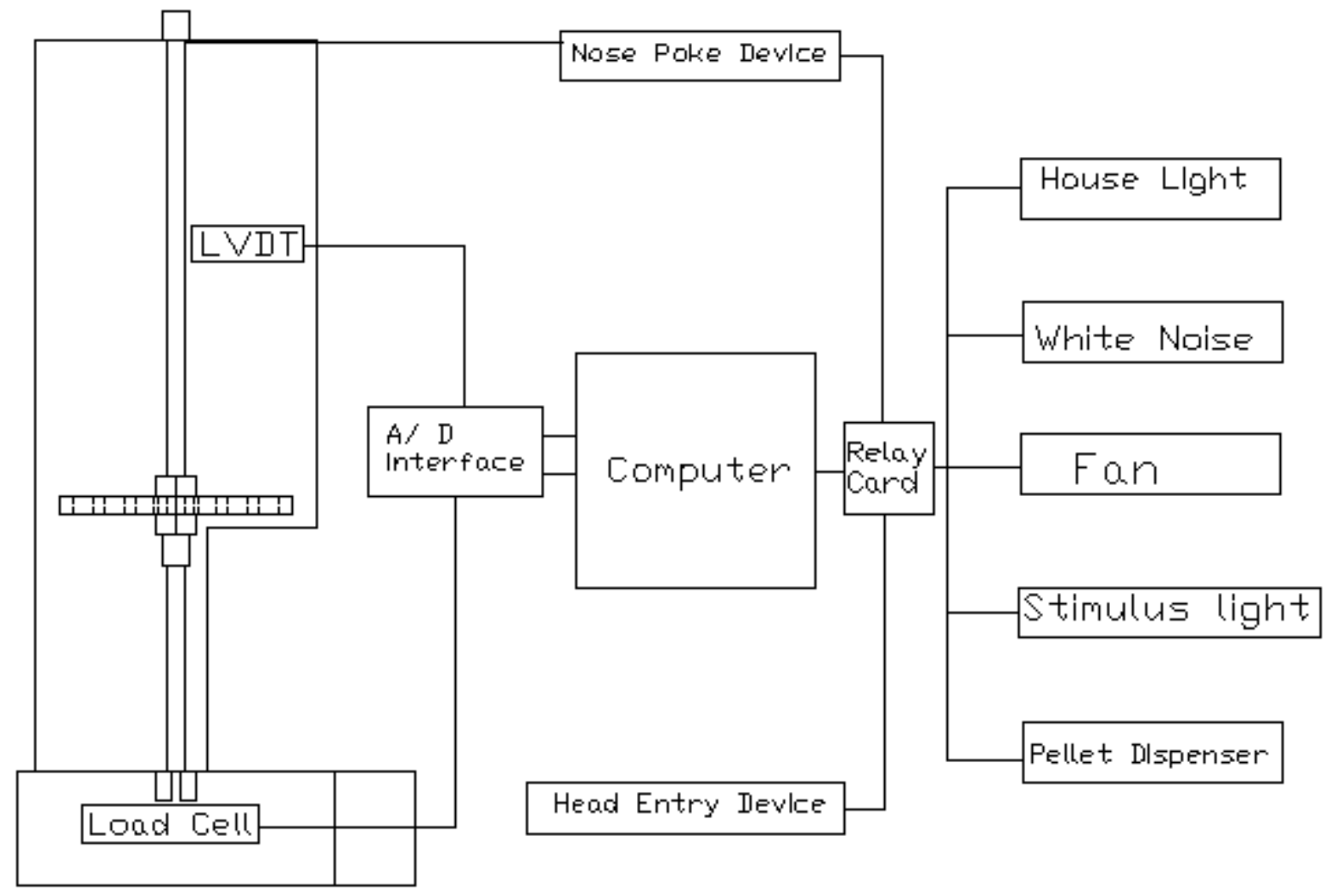

Figure 2- Schematic View of the Setup

\subsubsection{Overall Setup}

The overall setup shown in Figure 3 consists of a commercially available chamber placed within a noise attenuated cubicle (Med associates Inc,), a custom designed dynamometer, a Sensotec load cell, a 2310 signal conditioning amplifier, a Solartron Linear Variable Differential Transformer (LVDT), an OD4 signal conditioner compatible with the LVDT, a $333 \mathrm{MHz}$ computer and a Windows 98 platform.

The custom designed dynamometer is placed inside the test chamber, which measures $12^{\prime}$ ' $\mathrm{L}$ x 9 $1 / 2$ " W x 11 1/2" H. The inner portion of the cubicle measures 22" W x 15" H x 14" D. The dynamometer was designed to fit inside this cubicle along with the output devices as well as the displacement transducer because space was a premium. The dynamometer measures $5 \frac{1 / 2}{2}$ in width at the base and is about 10" in height. Figure 4 provides a close up of the apparatus with the cubicle denoted by (A), chamber marked as (B), the dynamometer base marked as (C), LVDT as (D), and the body of the dynamometer denoted by $(\mathrm{E})$. The external devices are marked as- Fan (F), nose poke device $(\mathrm{G})$, pellet dispenser $(\mathrm{H})$, and shields denoted as (I). 


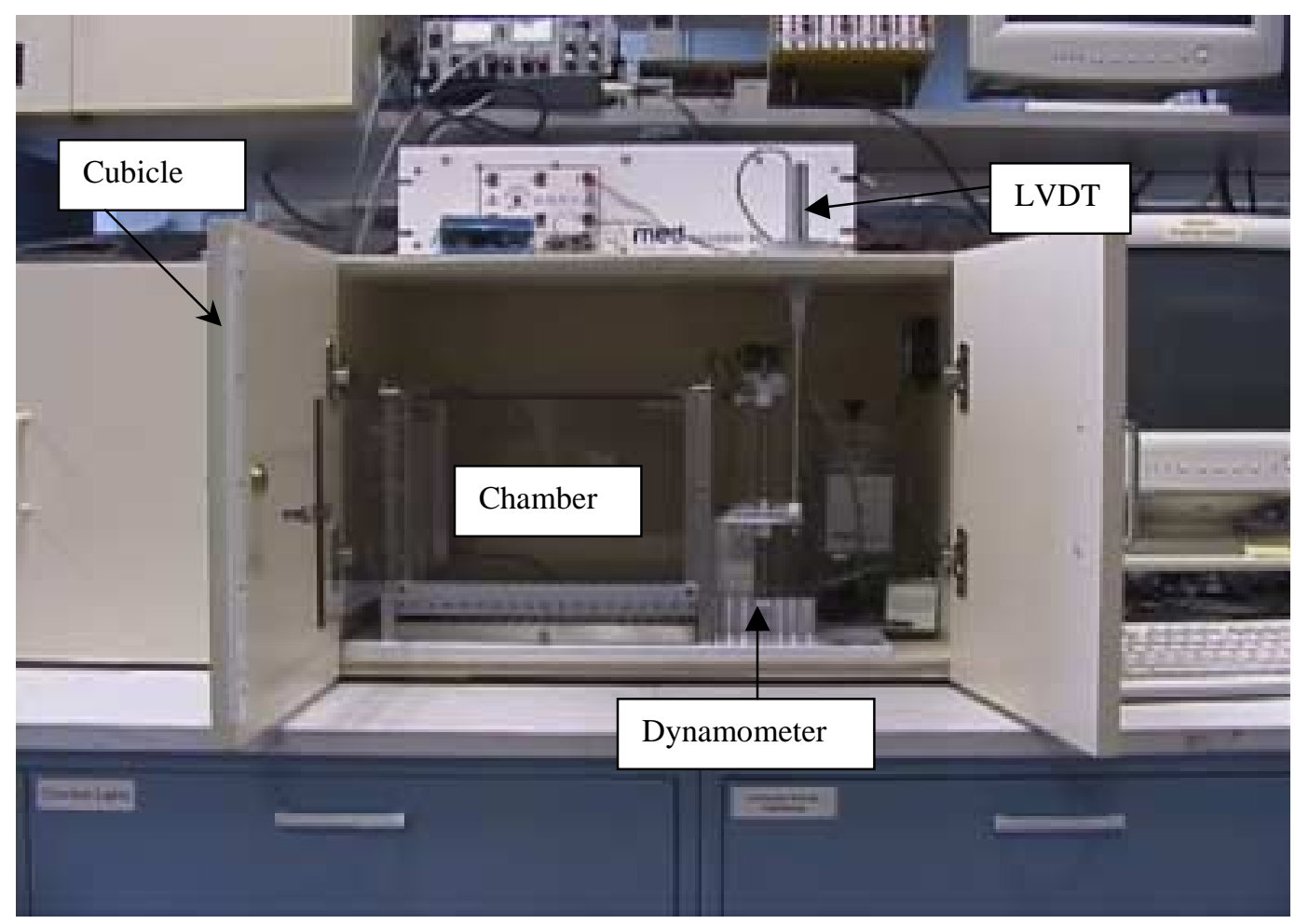

Figure 3 - Overall Setup

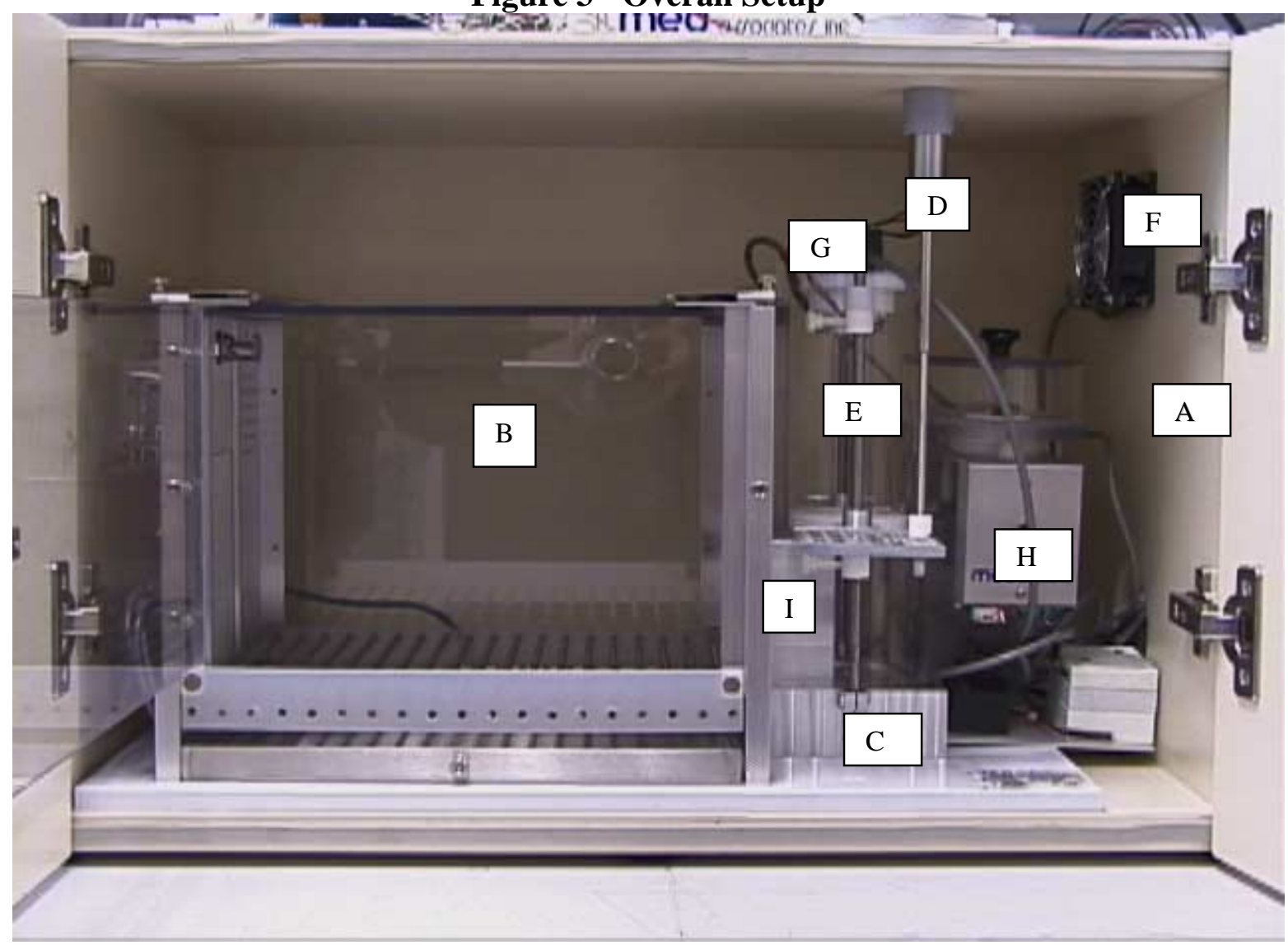

Figure 4- Set Up with the Transducer 


\subsubsection{System Overview}

\subsubsection{Base}

The base shown in Figure 5 is a solid rectangular block made of aluminum 6061 T651 measuring 4.5" wide, 1.5 " in height and 4.1" in length. The base is made from one solid block because it keeps the base in place within the chamber without having to screw it down to the floor of the cubicle thus making it accessible to be removed any time without difficulty, and it is easy to machine the part. A pocket of the shape denoted by (B) in Figure 5 is machined in the base to accommodate a force plate described in the next section. The pocket has four through holes marked as (C) machined along the vertical axis to accommodate the shafts of the force plate, which slide via linear bearings (Thomson Super 4 ball bushing bearings- closed type). A lip is provided within these holes to keep the bearings from sliding out. The open-ended holes allow the bearings to be pushed out from the bottom for lubrication or replacement. The bearings are snugly embedded in the base with an interference fit and held firmly in place using lock tite solution.

The base was fabricated to accommodate the Plexiglas tube alone. The shafts denoted by (D) support the yoke marked as (C) within the Plexiglas tube as seen in Figure 6, and projections or ears marked as (A) (refer Figure 5) were designed to keep them tightly in place. The projections have been split in the middle as seen in the front view of Figure 5 with provision for screws along the horizontal axis. Tightening these screws draws the ears closer and thus clamps the shafts in place securely.

The base pocket has another pocket marked (D) measuring 0.354" in diameter and 0.0019" deep, shown in Figure 5, machined in the center for the load cell. A rectangular slot (E) measuring 0.118" in width, 2.36" in length and 0.0011" deep is machined to bury the load cell wires and prevent the wires from being crushed by the weight of the force plate. The load cell pocket is made just deep enough so the load cell is flush with the surface of the base pocket. The load cell is snugly fit into the pocket with a press fit.

The base also accommodates shields that cover the gap above the base between the dynamometer and the opening of the chamber, preventing the rat from being distracted by external sights and sounds. These shields denoted by (I) can be seen in Figure 5. Three rectangular pieces measuring 1.57" x 3.149" $\mathrm{x} 0.236$ " were used to form the shield. The two vertical pieces were screwed to the base and the third piece was screwed to the top of these two pieces. This third piece was machined from a 1.57" x 2.952" x 0.118 " rectangular piece to suit the arc shape of the tube on one side. This projection on the other side is partially within the cubicle when the apparatus is set up and prevents the metal plates in the cubicle from sliding shut. 

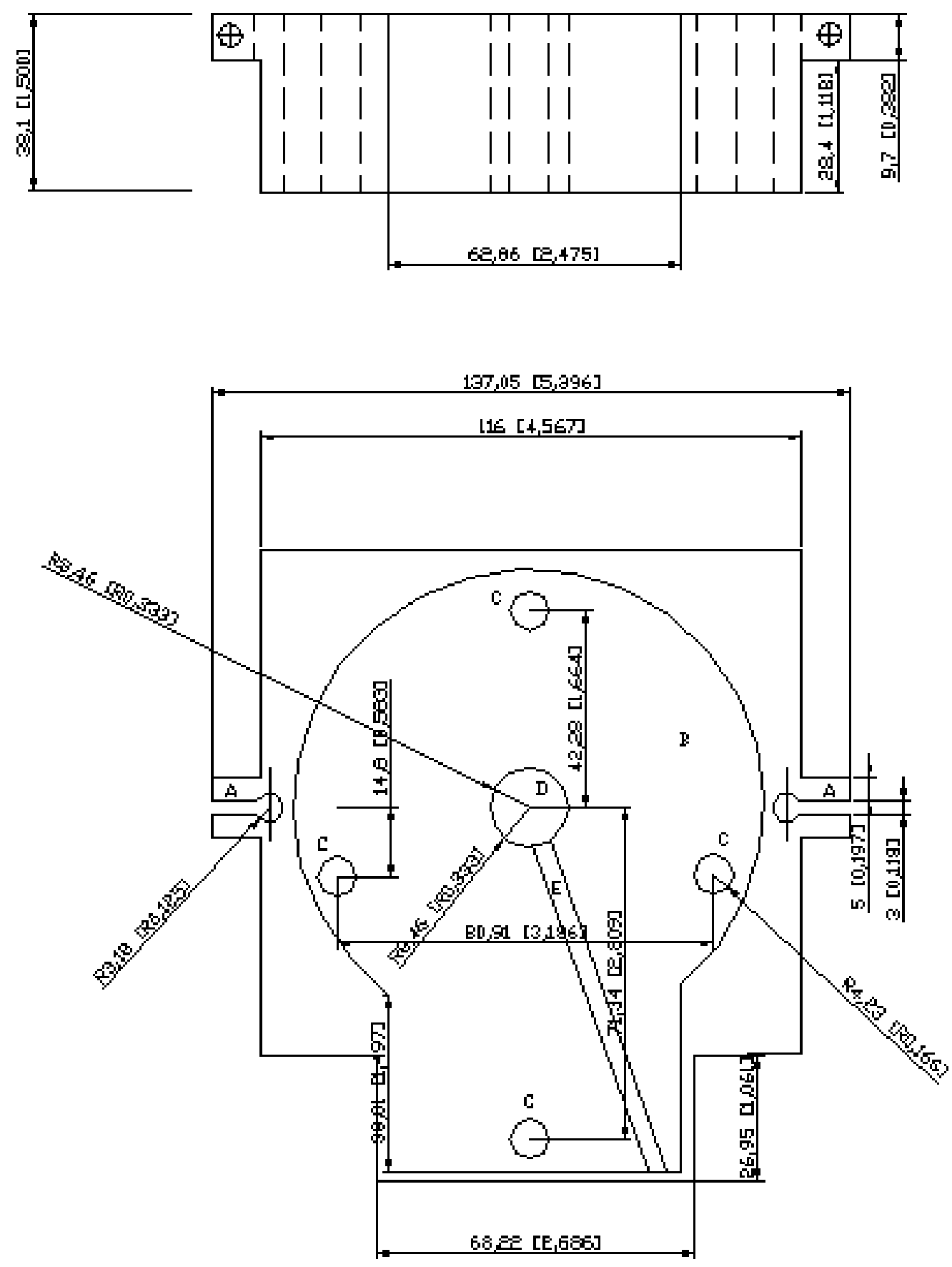

Figure 5- Front and Top Views of Base 

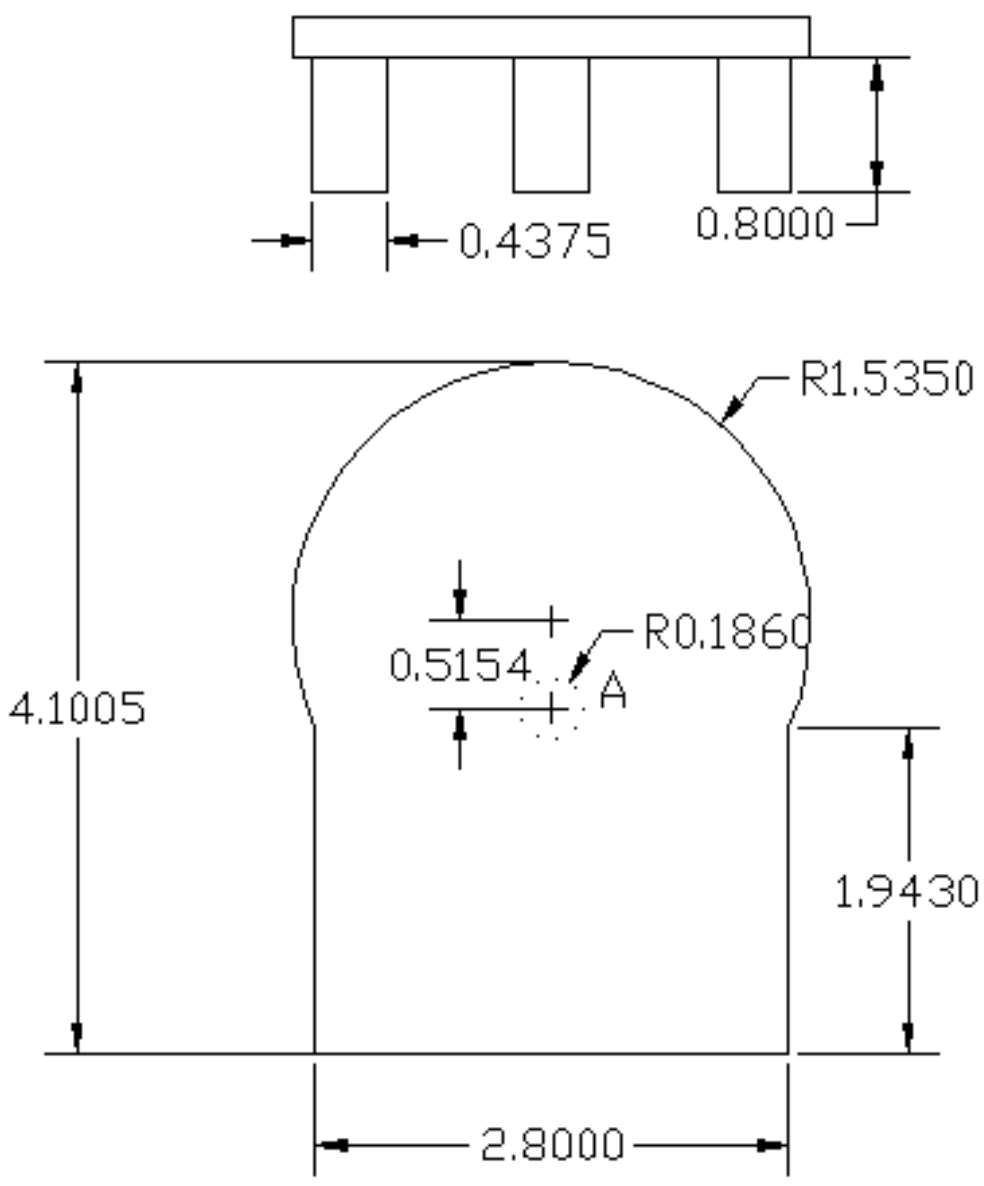

Figure 6- Front and Top Views of Force Plate

\subsubsection{Force Plate}

A force plate was machined to fit over the load cell and the pocket (Figure 6). The force plate has four $1 / 4$ " in diameter, class L shafts of length 0.8 " each with a hardness of 60 on a Rockwell C scale that have been set in the force plate using an interference fit and Lock Tite solution. The shafts slide along four Super 4 ball bushing linear bearings (Thompson Inc,) of closed type that are embedded in the base. The force plate has been designed to enable the load cell to detect even the slightest force generated by the rodent at the plantar surface of the foot. When the rat steps anywhere within the tube, the uniform linear motion of the force plate creates the same effect as the rat stepping on the load cell or the center of the force plate. Hence, the force plate is designed to cover the floor space within the tube. The base and the force plate are provided with a beak like projection which partially fits into the cubicle so that all gaps between the chamber and the dynamometer are completely closed when the opening of the chamber 
coincides with the opening of the dynamometer. This insures that the load cell picks up every force value generated by the rodent.

A button marked as (A), was provided on the underside of the force plate to coincide with the position of the load cell. When the force plate is placed in the pocket, the button rests on the load cell and the shafts stick out into the holes in the base. When the rat steps on the force plate, the button pushes down on the load cell sending out electrical signals proportional to the weight. Also the shaft and bearings allow for the downward movement of the force plate without any friction, thus enabling the apparatus to accurately detect the force values without the interference of any noise. To account for the weight of the force plate, the weight of the force plate including the shafts (147.57 $\mathrm{g}$ ) is subtracted from the final value of force.

\subsubsection{Plexiglas Tube}

The detailed view of the Plexiglas tube is shown in Figure 7. The tube is 12.5 " in height with an internal radius of 1.14 " and is 0.25 " thick. The tube is screwed down to the base from the bottom through two counter sunk holes. The Plexiglas tube denoted by A shown in the Figure 8 was designed to fit between the shafts and within the cubicle along with the transducer. The tube is used to restrict the movements of the rat during the lifting task. Figure 8 depicts isometric view of the dynamometer. The pans are denoted as (B), the Plexiglas tube as (A) and the yoke is marked (C).

The tube is open at both sides with an opening on one side measuring 2.75 " in width and 4 " in height. When the dynamometer is set up inside the cubicle this opening faces the opening of the chamber through which the rat can enter the tube from the chamber. The inside of the tube is flush with the shields described previously. The contours of the tube and shields coincide with the shape of the force plate. Slots have been cut along the sides of the tube to facilitate the sliding of the yoke and support, which extend out of the tube, along the shafts. On one side of the tube, the slot is wider at the top than at the bottom. This enables the support to be disengaged from the nose poke device and taken out without disassembling the nose poke device. 

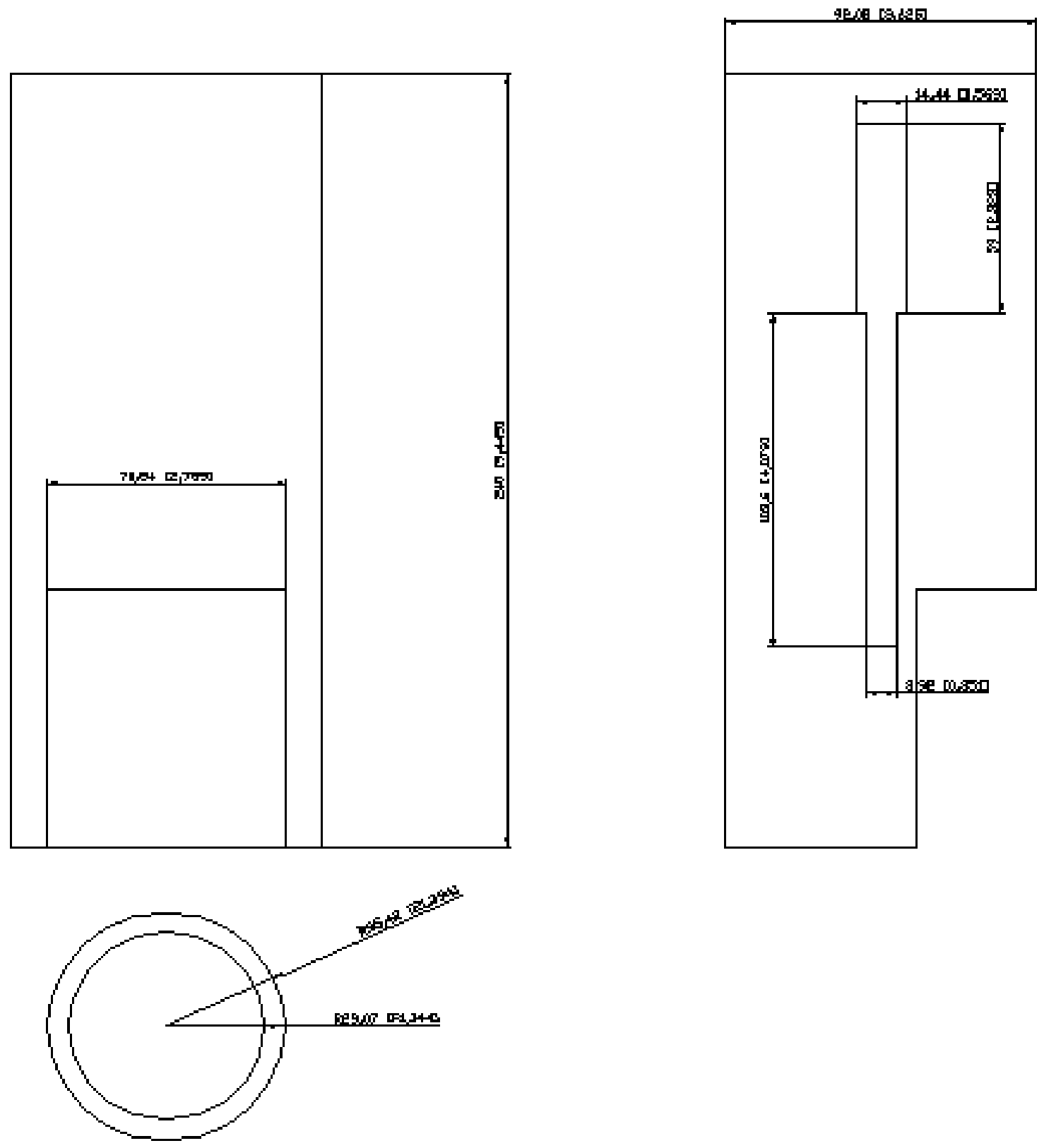

Figure 7- Front and Profile Views of the Plexiglas Tube 


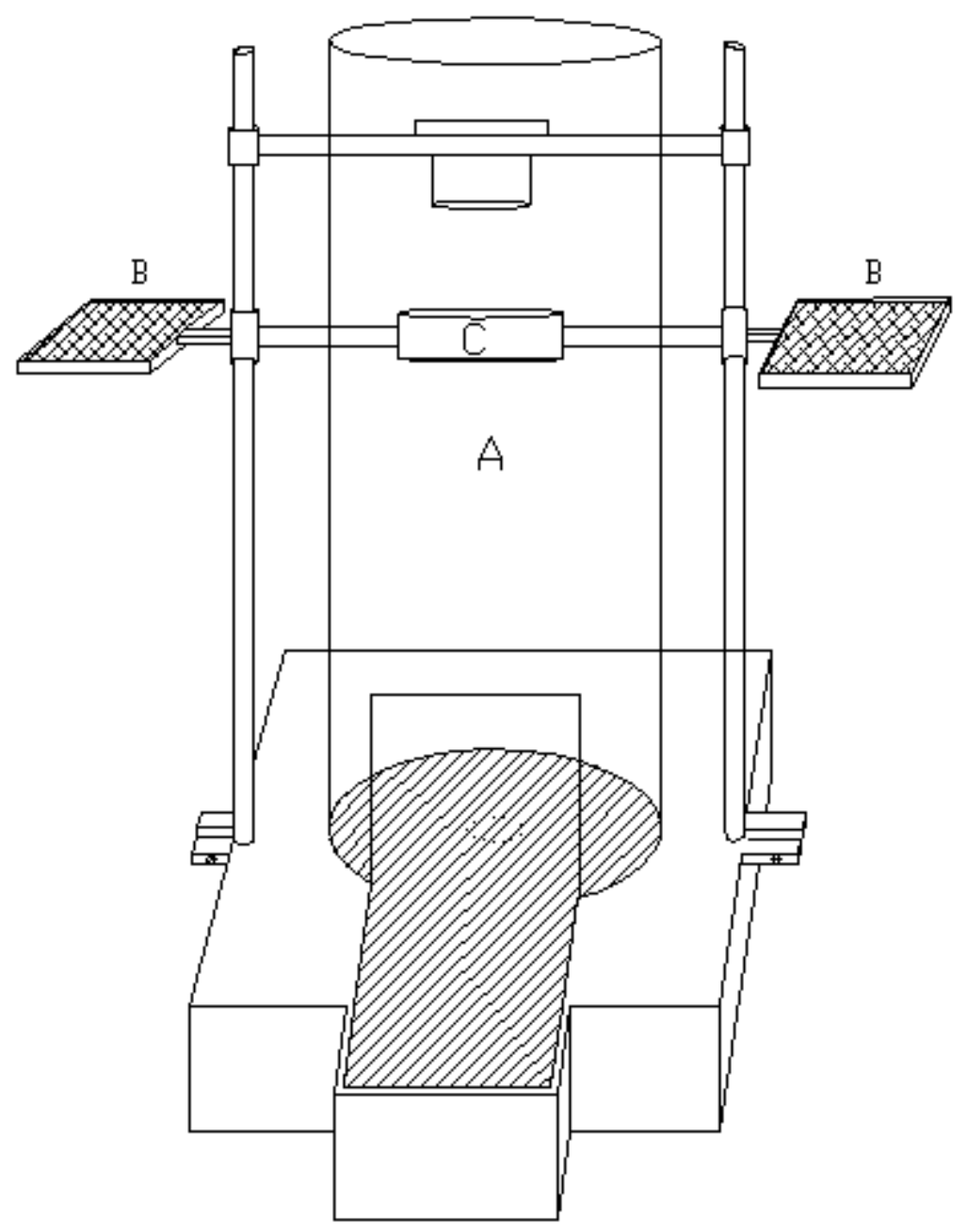

Figure 8- Isometric View of Dynamometer

\subsubsection{Pans}

Weight pans rectangular in shape machined from a flat piece of aluminum with a projection on one side and weigh approximately $74 \mathrm{~g}$ (Figure 9). The part marked (A) of the weight pan is a housing for the linear bearing. The housing projects and slides along the vertical shaft. A lip has been provided on the upper edge of the housing to prevent the bearing from sliding out. The bearing forms a snug fit to stay in place within the housing. The rectangular projection (B) that extends beyond the bearing housing into the Plexiglas tube is used to support the neck ring inside the tube.

To reduce the weight of the structure, symmetrical holes have been drilled in the pan. The weight pans have been constructed so that the load can be varied to change the biomechanical insults. As suggested earlier, injury can be induced in the muscles either by a repetition of the task or by varying the 
load. This represents on the job scenes when workers may have to lift heavy weights or perform repetitive tasks. The weight pans are designed to carry a maximum weight of 1000 grams. An edge is provided all around the structure to prevent the weights from being knocked over.

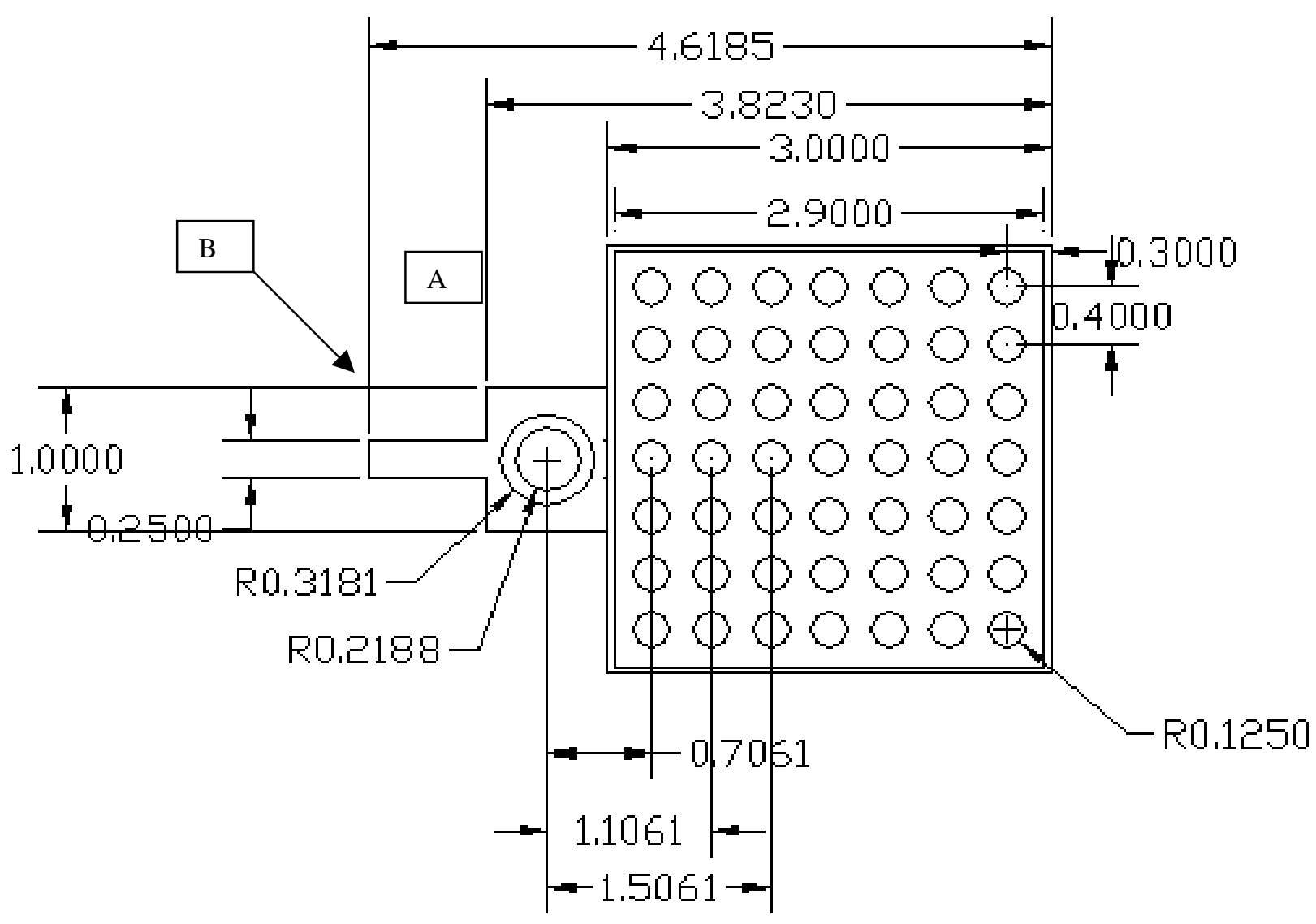

Figure 9- Top View of Weight Pan 


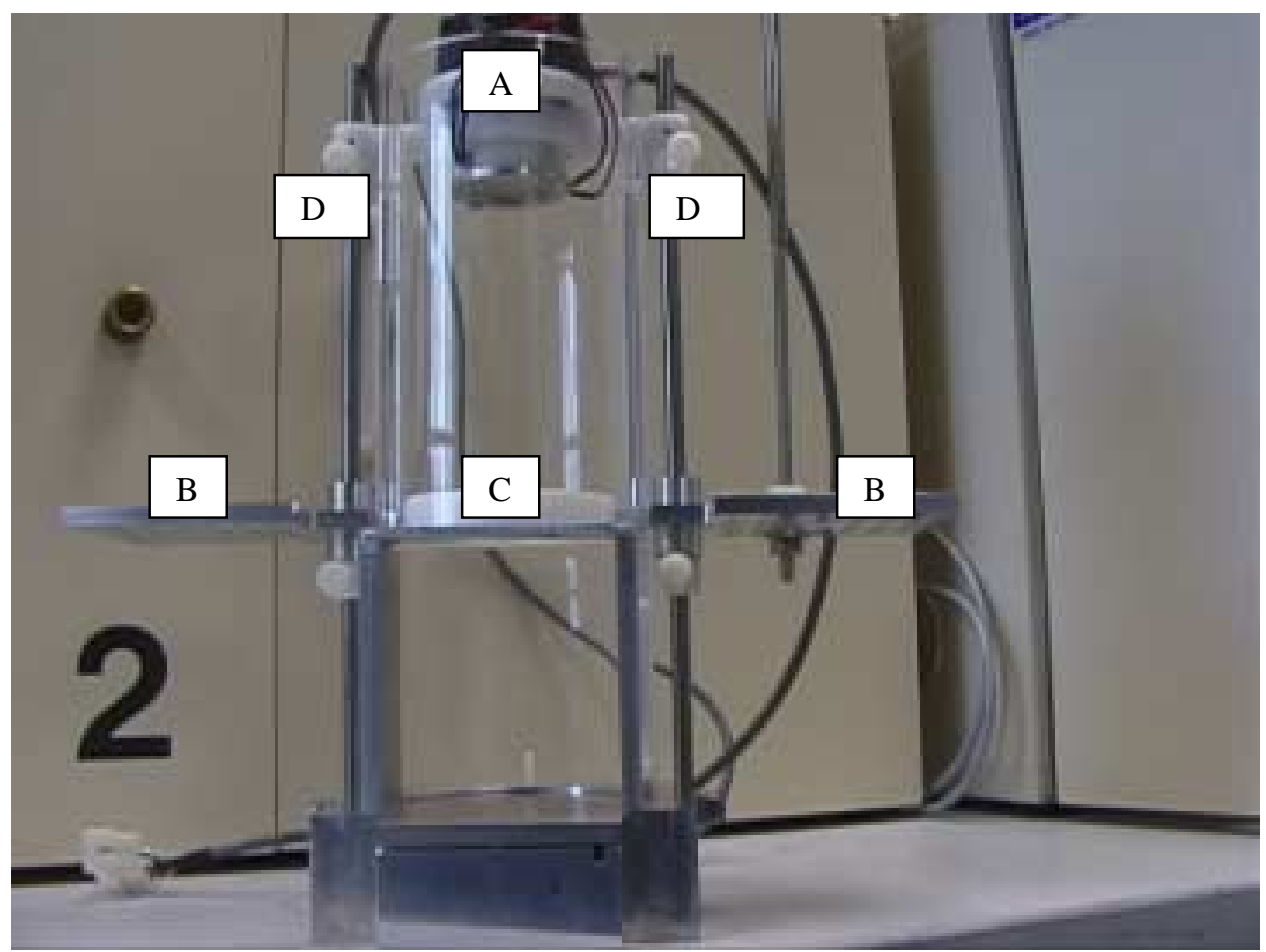

Figure 10- Front View of the dynamometer

\subsubsection{Neck Ring}

The neck ring, which is the centerpiece of the yoke, marked as $\mathrm{C}$ in Figures 10and 11, is made from Teflon due to its lightweight and ease of machinability. The neck ring is held in the center of the tube by the projections from the weight pans on either side. The neck ring and the weight pan assembly form a yoke that the rat is trained to lift. Square slots have been cut in the neck ring using a square broach to allow the weight pan projections to pass through and prevent the neck ring form spinning when the rat pokes or lifts the yoke. The length of the projection marked B in Figure 9 on the weight pan was designed so that, it would not protrude through the inner side of the ring and stab the rat in the neck even when the ring is pushed to one side. However, the projection had to be long enough to be able to hold the ring at the center preventing it from slipping out. The ring is designed as a separate piece so that different sizes can be machined according to the size of the rat being tested. 


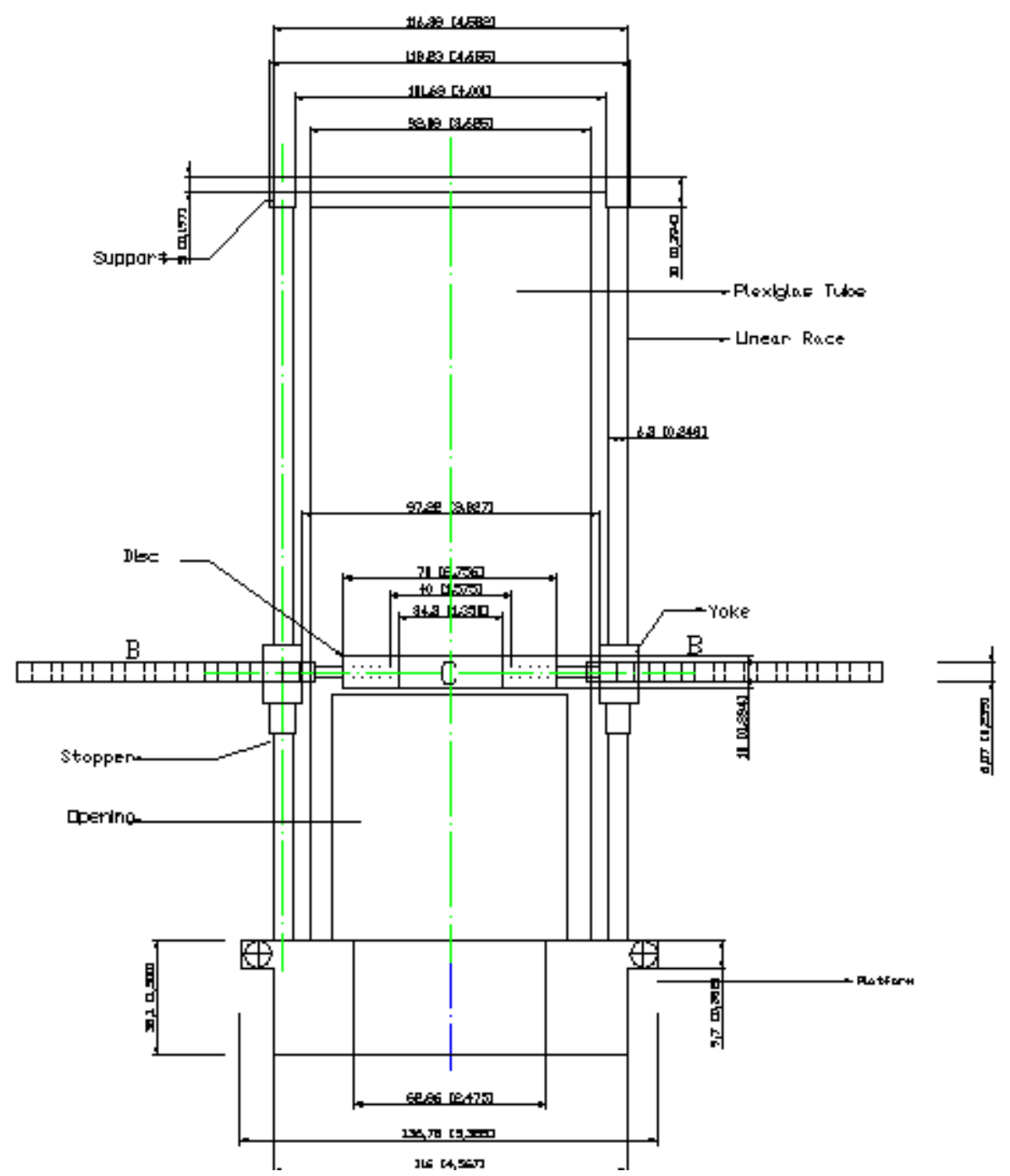

Figure 11- Front View of Dynamometer

\subsubsection{Support}

The vertical shafts, which measure 12.5 " in length, are held at the bottom of the base. The shafts need to be supported at the top to prevent them from keeling over. Hence a support as seen in Figure 12 made from Teflon was designed similar in shape to the yoke. The support is made of one piece with the ring's inner diameter measuring the same as the diameter of the nose poke device. Two holes on each end are drilled for the support to be able to slide along the vertical shafts. Also, being made of Teflon, the support does not damage the surface of the shaft along which the bearings slide, which is a critical parameter for a bearing surface. Threads have been tapped in these holes along the horizontal axis so that it can be tightened at different positions. Another important feature of the support is that it carries the nose poke device and stimulus light described later in this chapter. Provisions have been made to fix the 
support and thus the nose poke device at any desired height by tightening the support against the shafts using nylon screws. Fixing the support at a certain height allows the rat to be tested in the isometric mode. When the rat pushes up against the yoke fixed at a certain height, the muscles develop isometric force while trying to overcome the load.
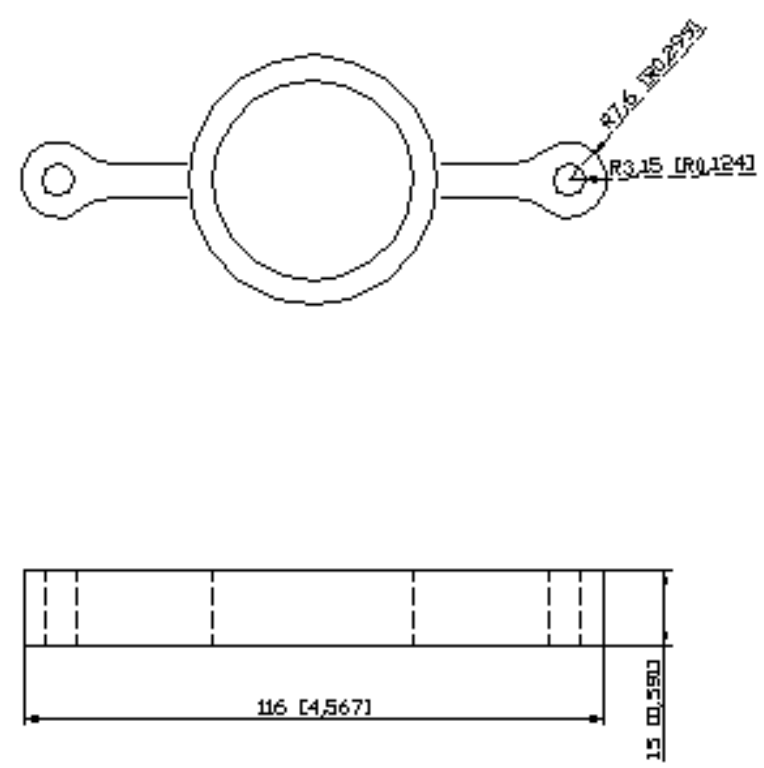

\section{Figure 12- Support for Shafts, Stimulus Light, and Nose Poke Device}

\subsubsection{Stoppers}

Stoppers made from Teflon are used to control the height of the yoke and the nose poke device and support assembly, which directly affects the range of movement of the lift. Two pairs of stoppers are used in the set up. The stoppers are open ended and cylindrical in shape approximately $1 / 2$ " in height with tapped holes on an axis perpendicular to that of their motion. Nylon screws are screwed in these holes to maintain them at a certain position. One stopper is used on each of the vertical shafts for the yoke and another pair for the nose poke device-support assembly.

\subsection{Hardware}

\subsubsection{Load Cell}

The load cell used for the system prototype is a Sensotec, model 13/2443-06. It has a range of 10 lbs. The load cell requires an excitation voltage of $5.0 \mathrm{~V}$ and produces a minute output of $2.1 \mathrm{mV} / \mathrm{V}$. The load cell as described previously is embedded in the base with the force plate placed on top of it. The load cell is connected to the computer via the A/ D board. The A/ D board is used to relay signals from 
the LabVIEW program to activate the load cell as well as relay back the data acquired to the computer. The load cell is also connected to a signal-conditioning amplifier. The amplifier is used to amplify the signal from the load cell to a value that can be comprehended by the $\mathrm{A} / \mathrm{D}$ board and the computer.

\subsubsection{Signal Conditioning Amplifier 2310 for the Load Cell}

The 2310 Signal Conditioning Amplifier provides conditioning and amplification of low-level signals from the load cell for display and recording on the computer. The 2310 SCA conditions and amplifies the signal from the Load Cell and relays the information to the (Computer Boards Inc, PCIMIO-16XE-10) A/D board. The Load Cell is connected to the SCA I/P channel. Power for the SCA is 105 to $125 \mathrm{~V}$ or 210 to $250 \mathrm{~V}$ (switch-selected), 50/60 Hz, 10 Watts, max.

For the system prototype, the excitation voltage was set at $5 \mathrm{~V}$ in order to drive the load cell, which requires 5.0 V DC. The gain is set at 203. From the gain equation,

$$
\mathrm{G}=\mathrm{V}_{\text {out }} / \mathrm{V}_{\text {in }}
$$

We can derive the input voltage of approximately $217.4 \mathrm{mV}$. The low-pass filter selection is set at $1 \mathrm{kHz}$. This establishes the cutoff frequency at $1 \mathrm{kHz}$. The filter allows all frequencies below this point to pass without attenuation while suppressing all frequencies above $1 \mathrm{kHz}$.

\subsection{Transducer Selection}

Transducers were selected based upon the type of core assembly and the maximum displacement measured. A guided type core assembly uses a spring return mechanism to push the core to its fully extended position. This type of mechanism would create a force (function of tension in the spring), which would work against the force generated by the muscle. The unguided core assembly type transducer allows the core to move freely without coming in contact the body.

\subsubsection{Ankle Mechanics}

A transducer was selected based on the maximum displacement to be measured. The dynamometer has been designed considering the largest rat that might be tested. The ankle mechanics had to be considered in selecting the stroke length of the transducer. From previous studies, the maximum angle of rotation for the ankle was found to be approximately $70 \mathrm{deg}$ and the distance from the point of application of the force was $22 \mathrm{~mm}$ approximately. This distance is on either sides of the joint about which the rotation takes place (Figure 13). Correspondingly, dx would be the maximum distance that the heel can be raised. 


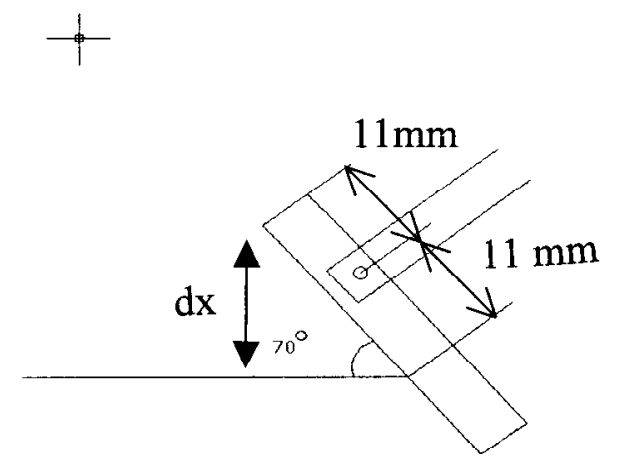

\section{Figure 13- Schematic of the ankle rotation (not to scale)}

Hence with a maximum ankle rotation of 70 deg the maximum distance of heel raise will be the value of $\mathrm{dx}$ calculated as follows

$$
\begin{aligned}
& \operatorname{Sin} 70=d x / 22 \mathrm{~mm} \\
& d x=20.6 \mathrm{~mm}
\end{aligned}
$$

Thus, the value of $\mathrm{dx}$ is $20.6 \mathrm{~mm}$. A transducer with a stroke length of $20.6 \mathrm{~mm}$ was needed. A 5 $\mathrm{kHz}$ mach 1 series, B25 Solartron transducer with a maximum stroke length of $25 \mathrm{~mm}$ was ideally suited for this purpose.

\subsubsection{Displacement Transducer}

The Solartron displacement transducer operates on the Linear Variable Differential Transformer (LVDT) principle. This principle states that movement of a core inside a transducer body is detected by a differential change in output on two secondary coils, the primary coils being energized by an appropriate $\mathrm{AC}$ signal. The subject rat's lifting the yoke displaces the probe causing movement of the inside core. Displacement is detected as a result of the difference in voltage potentials of the coils and this data is relayed to the computer (control center). The red and white connections are in phase for inward 
movement (i.e. towards the cable end). The output signal depends on both the core movement as well as the energizing voltage and is expressed as sensitivity in $\mathrm{mV}$ output/ $\mathrm{V}$ energizing/mm traveled.

The transducer's core is attached to the weight pans via lock nuts. The movement of the yoke will cause a displacement of the core within the transducer body thus causing a change in the voltage output. This voltage value is converted to a displacement value in terms of inches or centimeters by using an appropriate conversion factor.

\subsubsection{Transducer Sleeve}

A sleeve made of stainless steel was designed to connect the probe or the core of the transducer to the weight pan. The sleeve is threaded at one end and was shrink fitted to the bottom of the core without damaging its electrical properties. The threaded portion of the sleeve is inserted into one of the drilled holes on the pan and is held in place by using a lock nut. The sleeve also restricts unwanted movements of the transducer core to a minimum.

\subsubsection{Solartron Od4 Transducer Conditioner}

The OD4 unit contains the conditioning electronics to energize the Linear Variable Differential Transformer (LVDT) transducer and provide a DC output proportional to the displacement. The OD4 unit energizes the LVDT and provides a DC output to the PCI-MIO-16XE-10 A/D card (pins 34 and 68). Specifications are, unless otherwise noted, for a $5 \mathrm{~V}$ output into a $1 \mathrm{k} \Omega$ load with an input equivalent to a $200 \mathrm{mV}$ output from a transducer. The supply voltage for the OD4 unit is $10-30 \mathrm{~V}$ DC. The no load supply current is $140 \mathrm{~mA}$ maximum at $10 \mathrm{~V} \mathrm{DC}$ and $60 \mathrm{~mA}$ maximum at $30 \mathrm{~V} \mathrm{DC}$. The supply current given $20 \mathrm{~mA}$ output current is $180 \mathrm{~mA}$ maximum at $10 \mathrm{~V} \mathrm{DC}$ and $70 \mathrm{~mA}$ maximum at $30 \mathrm{~V} \mathrm{DC}$. The noise on the power supply is $20 \mathrm{mV}_{\mathrm{p}-\mathrm{p}}$ at $100 \mathrm{kHz}$ typical. Transducer energization is $3 \mathrm{~V} \mathrm{rms} \mathrm{sine} \mathrm{at} 5$ $\mathrm{kHz}$ with a transducer range of 45 to $450 \mathrm{mV} / \mathrm{V}$ full scale. The OD4 unit produces an output voltage of \pm $5 \mathrm{~V}$ DC full scale or $\pm 10 \mathrm{~V}$ DC full scale. The minimum load resistance is $1 \mathrm{k} \Omega$. The output current is \pm $20 \mathrm{~mA}$ full scales into $150 \Omega$ load maximum (only available with $5 \mathrm{~V}$ range selected). The output noise is less than $20 \mathrm{mV}_{\mathrm{p}-\mathrm{p}}$ at $10 \mathrm{kHz}$ or $100 \mathrm{kHz}$. For the system prototype, the OD4 unit is powered by a $+10 \mathrm{~V}$ voltage source and tied to common ground.

\subsubsection{Teflon Support For Transducer}

The total length of the transducer with the core at maximum stroke length is 7'. When the transducer is connected to the pan, the overall length exceeds the height of the cubicle. For this purpose a hole was drilled in the roof of the cubicle to accommodate the tail end of the transducer. To hold the 
transducer firmly in place, a flange made of Teflon was designed. The inner diameter of the flange is designed to be slightly smaller than the outer diameter of the transducer. When the transducer is slipped into the flange, the flange expands slightly and hugs the transducer tightly preventing it from slipping out.

\subsection{Software}

The program written using the LabVIEW software version $5.1[39,40,41]$ provided a graphic user interface to control various functions of the system. The user interface screen is shown in Figure 14.

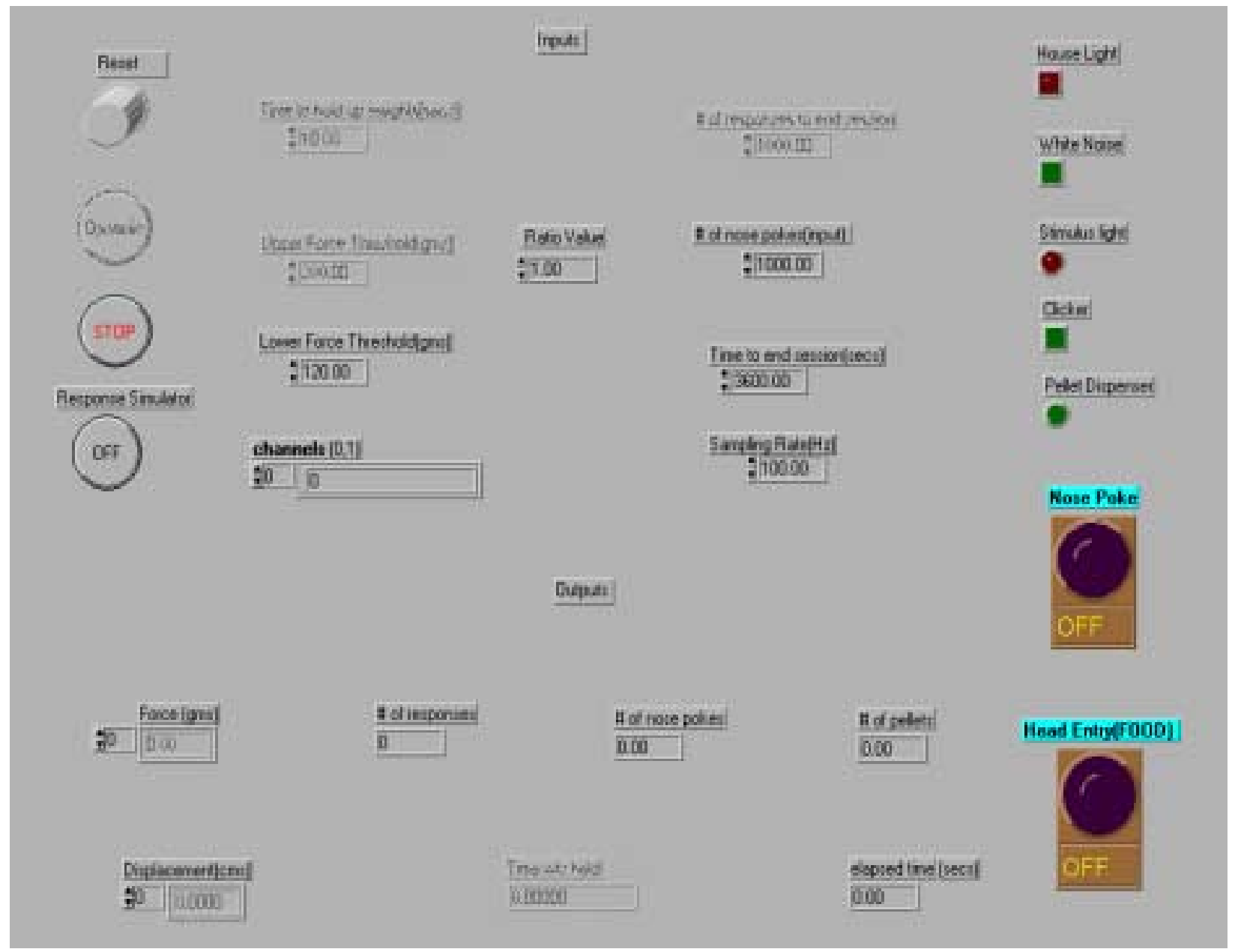

Figure 14- Front Panel or Graphic User Interface

The program is also capable of activating data acquisition of the transducers and control of devices. The input to the computer from the system comes from the nose poke and head entry infrared switch, allowing the program to count the number of "nose pokes", and from the load cell, allowing a display of the force generated in real time. 
As seen on the front panel, there are nine inputs along with the toggle button labeled "Dynamic". The user initially selects the dynamic or isometric mode of testing as desired. Depending on the mode selection the remaining inputs are entered.

In the case when the dynamic mode is selected, the "Time to hold up weights", and "Upper force threshold" inputs are disabled. The user only specifies the "Ratio Value", "Number of nose pokes to end session", "Lower force threshold", and "Time to end session". The number of nose pokes to end session and the time to end session determines when the session should be terminated. The lower force threshold determines whether a force value should be written to the database or not. Sending out values whenever the load cell is activated may result in the database to be voluminous, hence the lower force threshold filters out values lower than the value set by the user.

The stop button acts as a kill switch to abort the process at anytime during the session. The response simulator button is used to shape the responses of the rat during the initial training sessions. The button is used to reinforce successive approximation of the target lifting response. The reset button is pushed at the start of every session to clear previous entries.

The channels input is used to inform the computer and the data acquisition (DAQ) card about how many channels are being sampled. The sampling rate determines how many times the data should be read per second from the channels. Commonly, this value is set at $100 \mathrm{~Hz}$ implying that the load cell will be sampled 100 times a second or that the data on the spreadsheet would be separated by intervals of 0.01 second.

The lower portion of the front panel displays the output. The "Number of responses" indicates how many times the rat has been successful in breaking the infrared beam on the nose poke device. The "Number of pellets" which is a function of the ratio value and the number of successful responses indicates how many pellets have been rewarded. Usually, the number of nose pokes equals the number of responses. The elapsed time keeps track of time since start of the session. Elapsed time is continuously compared with the time to end session to terminate the session in progress. The force and displacement fields indicate the current values from the load cell and displacement transducer correspondingly.

In the isometric mode, the "Time weights held" field displays the time for which the rat held up the weights. A response would be considered satisfactorily completed when the user defined "Time to hold up weights" matches with "Time weights held" in the output field. The upper force threshold is used as a signal to start the timer to measure the time the weights were held. In other words, the lower force threshold is used as a cutoff to write force values to the database and the upper force threshold is used as a signal to start the timer. In this mode of testing, the force developed increases when the rat pushes up against the fixed yoke. When the rat pushes hard against the yoke, the plantar flexor muscles are directly affected. Thus rats are trained the to hold up the weights to investigate the isometric lifting performance. 
The indicators on the right display the status of the devices, which are out of sight of the user indicating exactly at what stage, the session is currently in.

\subsection{Computer System Interface}

The interface between the computer (the control center for the experiment) and the system is provided by the (Computer Boards Inc, PCI-PDISO16) input/output card. The Computer Boards Inc, PCI-PDISO16 is a 16-channel isolated input, 16-channel relay output interface board for PCI compatible computers. It is designed specifically for control and sensing applications. The board is interfaced with the computer via an ISA cable connected to ISA connection labeled P1 in the circuit diagram shown in Figure 15.

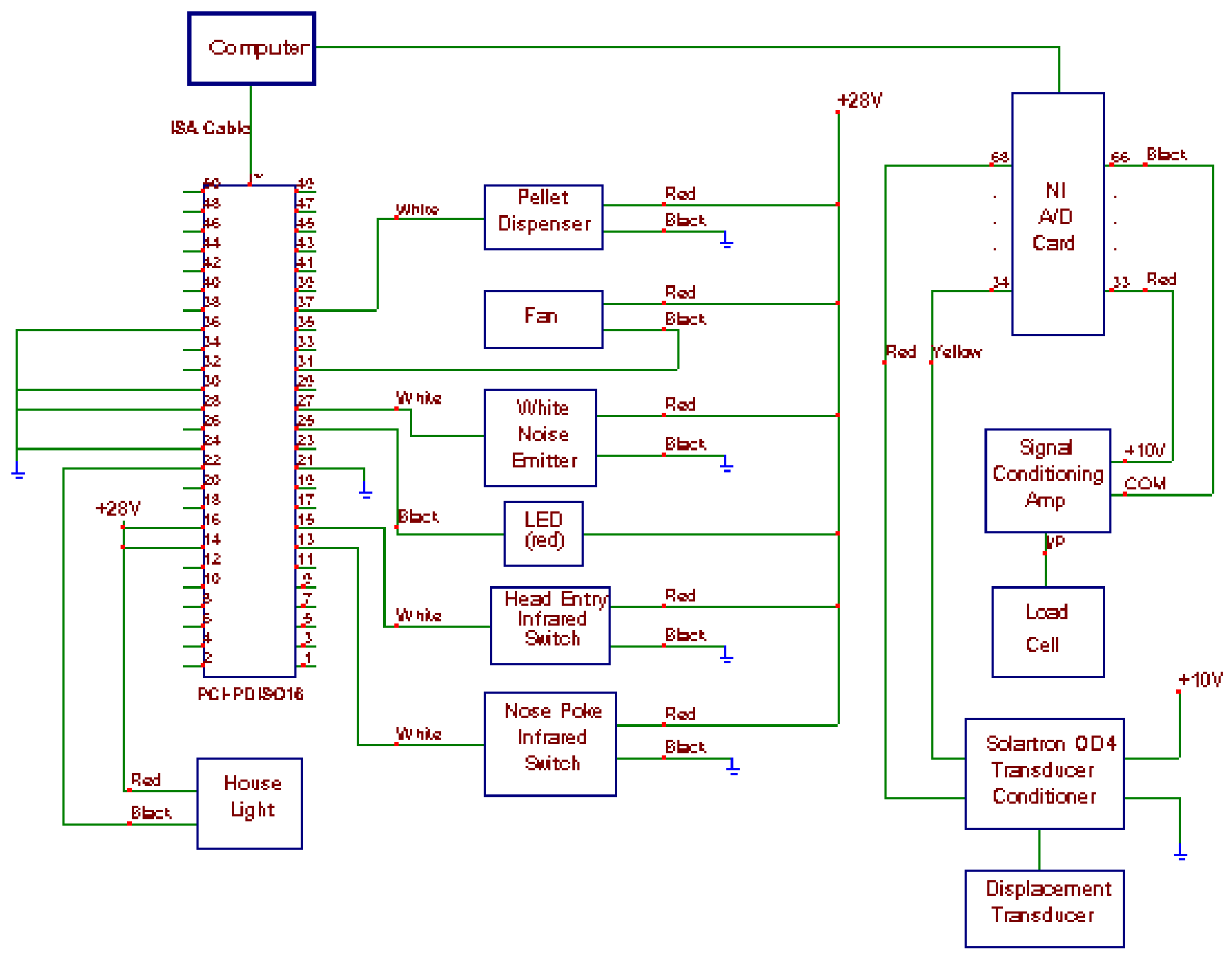

Figure 15- Wiring Diagram 


\subsubsection{Compute Boards Inc, PCI-MIO-16XE-10 A/D Board}

This A/D board is a high-performance multifunction analog, digital, and timing I/O board for PCI bus computers. The PCI-MIO-16XE-10 analog input circuitry can be calibrated for either a unipolar or bipolar polarity. Unipolar means that the input voltage range is between 0 and $V_{\text {ref, where }} V_{\text {ref }}$ is a positive reference voltage. Bipolar input means that the input voltage range is between $-\mathrm{V}_{\text {ref }} / 2$ and + $\mathrm{V}_{\text {ref }} / 2$. This board is a completely switchless and jumperless data acquisition board for the PCI bus. For the PCI-MIO-16XE-10 used in this set-up, for a range configuration of 0 to $+10 \mathrm{~V}$, the actual input range, given a gain of 1 , is 0 to $+10 \mathrm{~V}$. Pin 68 and pin 34 are connected to the Solartron OD4 Transducer Conditioner. Pin 68 (signal name: ACH0) is an analog input channel. (Note: Each channel pair can be configured as either one differential input or two single-ended inputs.) Pin 34 (signal name: ACH8) is also an analog input channel. Pin 66 is coupled to the COM connection on the Signal Conditioning Amp

The outputs are 16 FORM C electromechanical relays. The contacts are rated at $6 \mathrm{~A}$ at $120 \mathrm{~V} \mathrm{AC}$. or $28 \mathrm{~V} \mathrm{DC}$, resistive load. For the system prototype, output relay pin 37 was assigned to the Pellet Dispenser, o/p pin 31 to the House Fan, o/p pin 27 to the White Noise Emitter, o/p pin 25 to the LED (red), o/p pin 22 to the House Light, and o/p pins 36, 30, 28, 24, and 21 are connected to a common ground.

There are 16 individual, optically isolated at $500 \mathrm{~V}$, inputs that may be read back as two 8-bit bytes. These inputs are not polarity sensitive, so they can be driven by either an AC. voltage at $50-1000$ $\mathrm{Hz}$ and $5 \mathrm{~V}-24 \mathrm{~V}$, or by a DC signal at $5 \mathrm{~V}-24 \mathrm{~V}$. Each individual input has a software-enabled low-pass filter with a time constant of $5 \mathrm{mS}$ at $200 \mathrm{~Hz}$. The input filters are described and set by InstaCAL. InstaCAL is the installation, calibration, and test software provided with the PCI-PDISO16 I/O board. For the system prototype, input pin 13 was assigned to the Head Entry Infrared Switch and input pin 14 was connected to $+28 \mathrm{~V} \mathrm{DC}$ and pin 33 is wired to the $+10 \mathrm{~V}$ connection on the Signal Conditioning Amp. Pin 66 (ACH9) and pin 33 (ACH1) are both analog input channels. The PCI-MIO-16XE-10 should have DC input coupling and each input should remain within $\pm 11 \mathrm{~V}$ of ground.

The PCI-MIO-16XE-10 has 2 voltage output channels with a resolution of 16 bits, 1 in 65,536, and a maximum update rate of $100 \mathrm{kS} / \mathrm{s}$. It is a double-buffered DAC with FIFO buffer size of 2,048 S. Types of data transfers include DMA, interrupts, and programmed I/O. It has a relative transfer accuracy (INL) of $\pm 0.5 \mathrm{LSB}$ typical, and \pm 1 LSB maximum. The board has a voltage output of $\pm 10 \mathrm{~V}, 0$ to $10 \mathrm{~V}$ (software selectable). Output coupling should be DC. Current drive is given as $\pm 5 \mathrm{~mA}$. The board has 8 digital input/output channels that are TTL/CMOS compatible. Digital logic levels are as follows: input low voltage minimum: $0 \mathrm{~V}$, input low voltage maximum: $0.8 \mathrm{~V}$, input high voltage minimum: $2 \mathrm{~V}$, input high voltage maximum: $5 \mathrm{~V}$, output low voltage maximum: $0.4 \mathrm{~V}$, output high voltage minimum: $4.35 \mathrm{~V}$. 
The PCI-MIO-16XE-10 interfaces with the computer (the control center) via a PCI connection. The wiring diagram is shown in Figure 15.

\subsection{Accessory Devices}

\subsubsection{Pellet Dispenser}

The pellet dispenser is used to reward the rat for accomplishing the specified physical task of lifting weights. The dispenser is powered by the $28 \mathrm{~V}$ DC power supply and is connected to common ground. The "activate" wire, in this case, the white one, is connected to pin 37 of the PCI-PDISO16 input/output interface card. When a user-defined response is met, the LabVIEW program sends a signal to the relay card, which pulses the pellet dispenser.

\subsubsection{L- Frame Support for the pellet dispenser}

Floor space being a premium, the pellet dispenser was not used in the position it was originally built for by the manufacturer. Hence, to raise the pellet dispenser to the height built by the manufacturer at which functions optimally, an L shaped frame was designed to support the device. With this new design, the height at which the pellet dispenser is held can be varied for optimum performance. The Lframe was made from aluminum as shown in Figure 16.

Changing the design of the pellet dispenser, the original feeder tube had to be replaced by a longer tube to deliver the food pellets into the food trough. The height of the pellet dispenser and the delivery tube are important factors because a wrong selection can cause the food pellet to shoot out of the trough or may end up not being delivered in the shortest time interval.

\subsubsection{Fan}

The fan provides ventilation for the house. It is powered by the $28 \mathrm{~V}$ DC power supply. The fan is connected to pin 31 of the PCI-PDISO16 interface card. This is the "activate" wire for the fan. The fan is turned on immediately when the program is executed and remains in the "on" position until the termination of the program.

\subsubsection{White Noise Emitter}

The White Noise Emitter produces white noise within the house to block out any external noise that might distract the rat from its task. The emitter is also powered by the $28 \mathrm{~V} \mathrm{DC}$ power supply and is tied to common ground. The white "activate" wire is connected to pin 27 of the PCI-PDISO16 card. 
The white noise is turned on when the program is first executed and is offset to correlate it with the reinforcement. The white noise is offset by a 100-millisecond interval. This distinctly signals the rat to go and get its food. By turning the white noise on and off, the rat learns to correlate the offset of the white noise with the reinforcement.

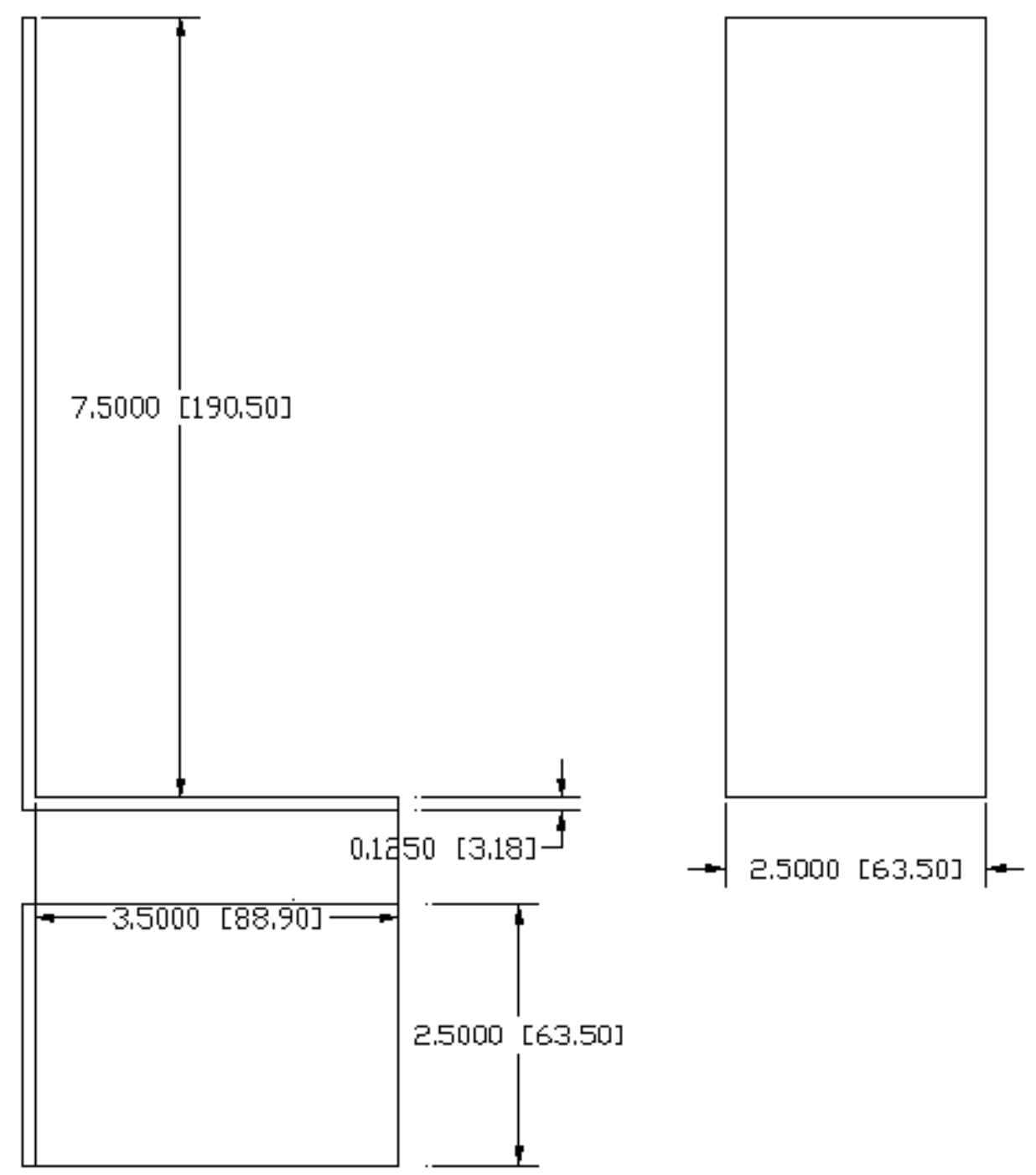

Figure 16- Top, Profile and Front Views of the L-Frame

\subsubsection{Light Emitting Diode}

Turning on the stimulus light, signals the subject rat to perform the specified task. The LED is powered by the 28V DC supply. Its "activate" wire is connected to pin 25 of the PCI-PDISO16 board.

The stimulus light is turned on at the beginning of the execution of the program and is turned off to correlate the offset with the reinforcement. The stimulus light is turned back on when the rat retrieves 
the food pellet. This continues until the end of the session. The stimulus light along with the white noise and a food reward strengthens the lifting response shaping of the rat.

\subsubsection{Head Entry Infrared Switch}

The head entry infrared switch informs the system when the rat has retrieved the food pellet. The rat's snout breaks the infrared beam, thereby engaging a switch and closing the circuit. Data is relayed back to the computer. The switch is powered by the $28 \mathrm{~V}$ DC power supply and tied to common ground. Its "activate" wire (white) is connected to pin 15 of the PCI-PDISO16 interface card. The program has been designed such that, the session does not proceed until the rat has retrieved the food pellet from the trough. This ensures that the rat is retrieves the food pellet.

\subsubsection{Nose Poke Infrared Switch}

The nose poke infrared switch informs the system of the number of lifting repetitions the subject rat has performed. The rat's snout breaks the infrared beam, thereby engaging the switch and closing the circuit. The data is relayed back to the computer. The nose poke infrared switch is powered by the $28 \mathrm{~V}$ DC power supply and tied to common ground. Its "activate" wire (white) is connected to pin 13 of the PCI-PDISO16 board.

This device functions identical in mechanism to the head entry device. There is a continuous infrared beam in the nose poke device. When the rat lifts up the yoke assembly all the way to the top, the rat's snout breaks the infrared beam in the nose poke device signaling to the rat that the lift is complete. The breaking of this beam is considered a response in the dynamic mode. This immediately signals the program to turn off the white noise momentarily, turn off the stimulus light, activate the feeder and wait for the infrared beam in head entry device to be broken to proceed.

\subsubsection{House Light}

The house light provides illumination inside the house. It is powered by the $28 \mathrm{~V}$ DC supply and is connected to pin 22 of the PCI-PDISO16 card for activation purposes. The house light is turned on at the start of the session and remains on until the session is terminated.

\subsection{Triple Output Power Supply}

The triple output power supply operates as three separate power supplies. Each has floating output and is completely isolated from the other two. One is a fixed 5V supply while the other two (A and B) are continuously variable over a 0 to $24 \mathrm{~V}$ range. For the system purposes, either supply A or 
supply B is set to $10 \mathrm{~V}$. This $10 \mathrm{~V}$ is used to power and ground the Solartron OD4 Transducer Conditioner.

\subsection{Nose Poke Fixture}

The commercially available nose poke response device from Med Associates, Inc was redesigned to fit within the Plexiglas tube supported by the vertical shafts. The 3 piece rectangular design was changed to a two-piece cylindrical design made from Teflon. The nose poke device as shown in Figure 15 was constructed to be held at the center of the tube with the LED and infrared beam side facing the base. The support described earlier is designed to carry the nose poke device. The support shown in Figure 12 has a ring in the center into which the nose poke device is inserted and screwed on tightly.

The nose poke device has an open ended cylindrical piece about $0.5 \mathrm{cms}$ in height with a lip inserted into the support. On top of this tube, the printed circuit board (PCB) is placed which is covered with a cap to protect it from damage. Also, this cap carries the infrared beam device. A slot is provided in the cap for the wires. Two screws long enough to hold all the pieces including the infrared beam device together are used to screw them together. At the bottom of this fixture, which is the side facing down the tube, is an aluminum ring, which is used to create the same effect as on the original Med Associates device which was used to train the rat.

The nose poke device has to be assembled inside the tube because of its complex design. Once assembled inside and screwed down to the support, the support and the nose poke device moves along the shafts as one assembly. 

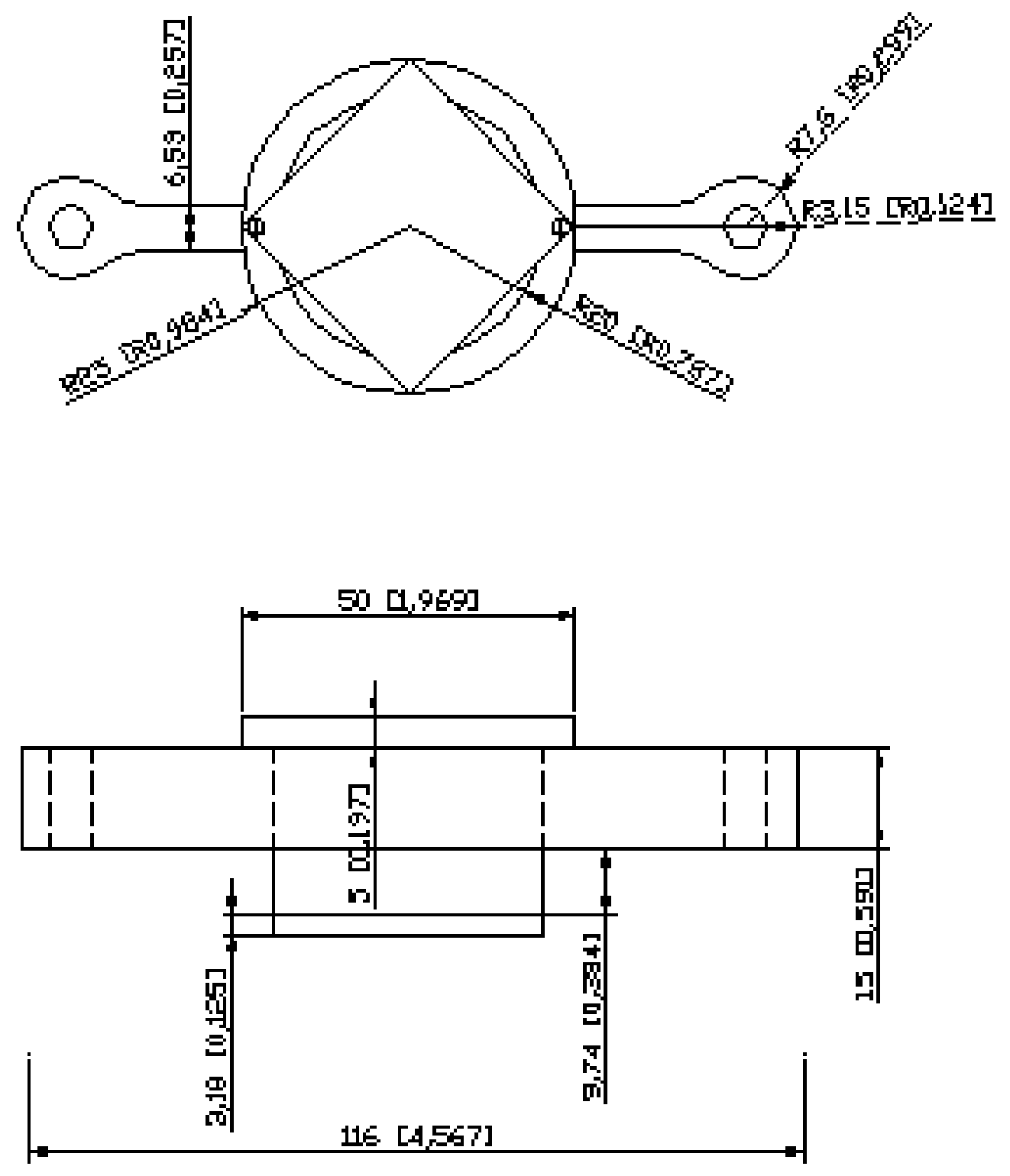

Figure 17- Modified Nose Poke Device With the Support 


\section{CHAPTER 3}

\section{CALIBRATION AND TESTING}

\subsection{Transducer and Signal Conditioner}

The transducer and the OD4 were first set to a mechanical and electrical null (or zero) by using the offset control, the full-scale output was then set by using the span control on the conditioner. The transducer was unplugged and a short circuit was applied across pins 4 and 5 of the DIN socket. The offset control was adjusted until zero was displayed on the instrumentation. The short circuit was then removed and the transducer plugged back in. The body was adjusted until zero was again displayed on the instrumentation. Then the conditioner was set to give a correct reading for any particular transducer displacement.

The transducer was moved to a known position and the span control was adjusted until the instrumentation showed the required reading. The transducer has a sensitivity of $16 \mathrm{mV} / \mathrm{V} / \mathrm{mm}$. The voltage range was selected to be $0-10$ volts. Hence for a maximum stroke length of $2.5 \mathrm{cms}$ the voltage output would be 10 volts and for no displacement the display on the instrumentation would be 0 . The voltage readings were then converted to displacement in terms of centimeters using an appropriate conversion factor to verify the accuracy of the instrument. Table 1 contains the calibration data collected for the LVDT. Figure 18 shows

Table 1: LVDT Calibration Data

\begin{tabular}{|c|c|}
\hline Voltage (Volts) & Distance (cms) \\
\hline 0.49 & 1 \\
\hline 1.201 & 3 \\
\hline 2.2 & 5 \\
\hline 4.1 & 10 \\
\hline 4.7 & 12 \\
\hline 6.01 & 15 \\
\hline 7.15 & 18 \\
\hline 7.9 & 20 \\
\hline 8.7 & 22 \\
\hline 10.1 & 25 \\
\hline
\end{tabular}




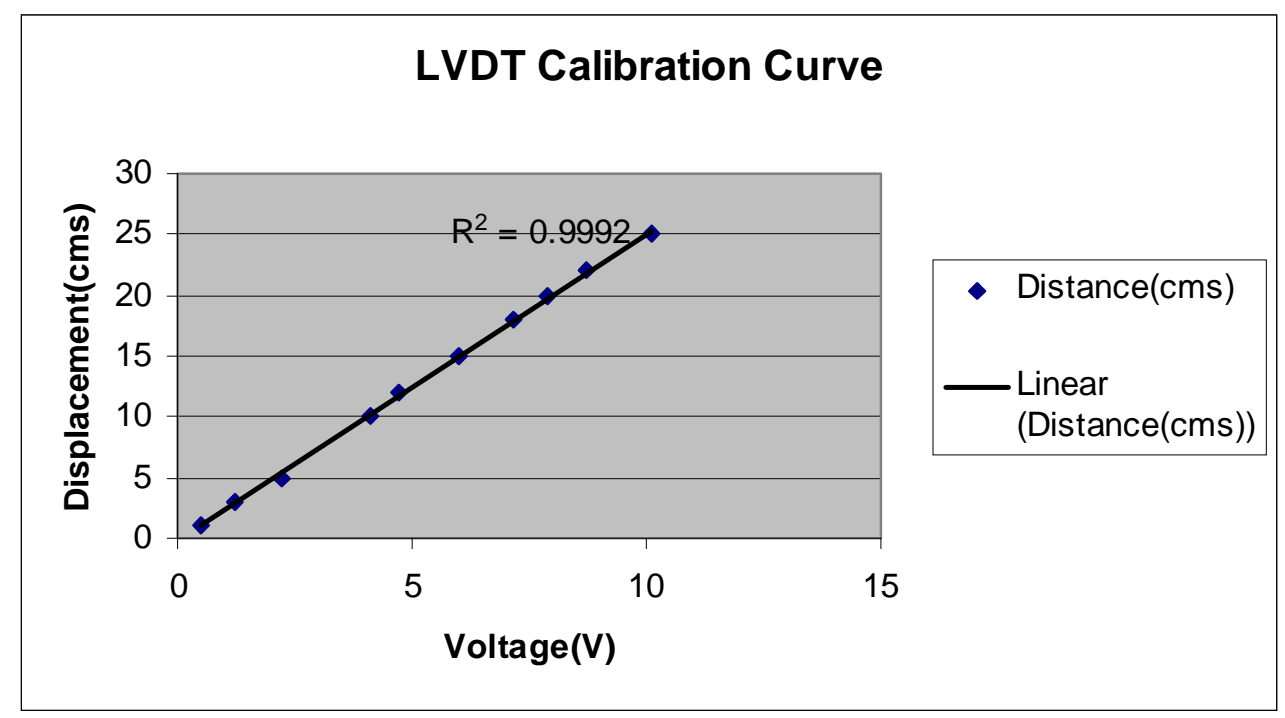

Figure 18 - LVDT Calibration Curve

\subsection{Load Cell}

The load cell puts out a maximum value of $2.1 \mathrm{mV} / \mathrm{V}$ for $10 \mathrm{lbs}$. Once the load cell was set in the base and the force plate placed over it, precision weights ranging from 100 grams to a 1000 grams were placed over the plate and the voltage output was recorded. Plotting these values as shown in Figure 19 and performing a regression analysis, the r-squared value was determined to be 0.999 . The following table illustrates the corresponding voltage values for the different precision weights. These voltage readings were converted to load in terms of pounds using a correction factor. The data can be found in Table 2.

Table 2 Load Cell Calibration Data

\begin{tabular}{|c|c|}
\hline Load (Grams) & Voltage (Volts) \\
\hline 100 & 0.0452 \\
\hline 200 & 0.0940 \\
\hline 300 & 0.1400 \\
\hline 500 & 0.2250 \\
\hline 1000 & 0.4620 \\
\hline 4545 & 2.0000 \\
\hline
\end{tabular}


Loadcell Calibration Curve

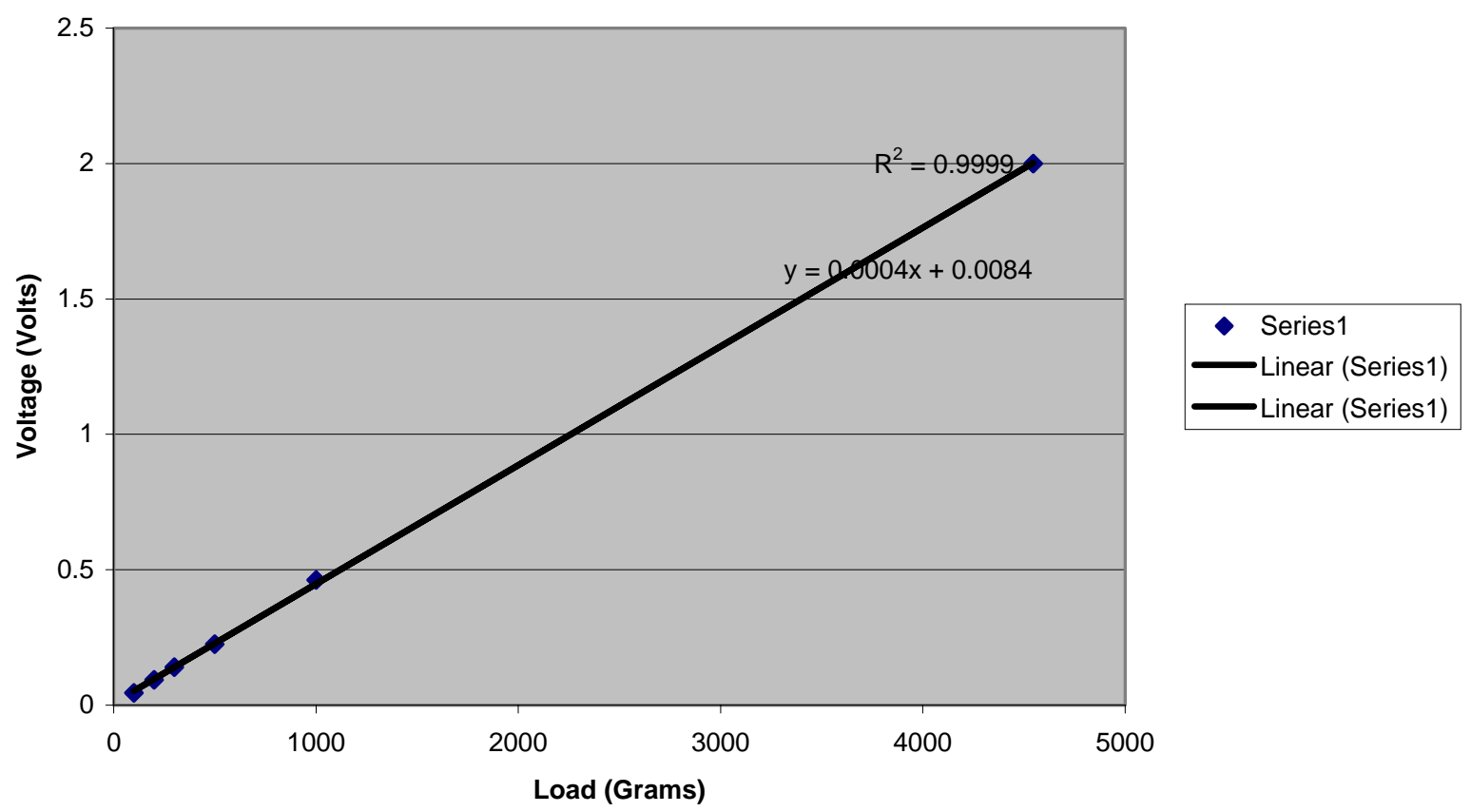

Figure 19. Load Cell Calibration Curve

\subsection{Operant Conditioning}

The rat was first trained to respond to the stimulus light using a commercially available nose poke device. The nose poke device was placed inside the cubicle with the LED and infrared beam facing the chamber so the rat could insert its snout in the device. A software program using LabVIEW 5.1 was developed to automate the training process. Food pellets are used as incentives to train the rat to respond to the stimulus light.

\subsubsection{Pseudocode for the operant conditioning software:}

1. Start white noise, house light, and stimulus light.

2. If infrared beam in nose poke device is broken.
a. Offset white noise with a 100-millisecond interval
b. Turn off stimulus light.
c. Activate feeder
d. Dispense pellet.

i. If infrared beam in the food trough is broken.

1. Turn stimulus light back on and proceed with session. 
ii. Else

1. Wait

3. Else

a. Wait.

The white noise, house light, and the fan are turned on when the training session begins. Initially, the user sets the ratio value, number of nose pokes to end the session and also the time to end the session. The ratio value is used to determine after how many nose pokes or responses a food pellet is to be rewarded. The number of nose pokes and the time to end the session criteria determines when the session should end, whichever occurs first.

Once the values are set and the program is activated the user does not intervene until the session ends. Given its explorative nature, the food-deprived rat will go around the cage exploring the gaps and holes. Consequently, when the rat trips the infrared beam in the nose poke device, the stimulus light and white noise go off and at the same time the pellet dispenser releases a food pellet. The rat begins to correlate the reward with the switching off of the white noise for a 100-milliseconds and the stimulus light not being turned back on until the rat has retrieved its food pellet. Another infrared beam has been set up in the trough where the food is delivered from the dispenser. When the rat retrieves the food pellet from the tray, it trips the beam thus sending a signal to the software to proceed with the training session.

When the program is first executed, the DAQ card continuously samples the signal to see if the nose poke infrared beam has been broken. When the rat trips the infrared beam, it closes the switch of the device, thus sending out a voltage signal. This signal is relayed back to the computer through a relay card. The software then activates the pellet dispenser, white noise and stimulus light by sending out signals through the relay card. The two inputs (nose poke device and the head entry device) and five output devices (the white noise, house light, stimulus light, the pellet dispenser, and the fan) are connected to the relay card.

\subsection{Testing}

\section{Notations used in the flowcharts and pseudocodes:}

$$
\begin{aligned}
& \text { To }=\text { Elapsed Time } \\
& \text { T1 }=\text { Time to hold up weights } \\
& \text { T2 }=\text { Time weights held } \\
& \text { T3 }=\text { Time to end session } \\
& \text { R0 }=\text { Number of responses to end session } \\
& \text { R1 }=\text { Number of responses }
\end{aligned}
$$


$\mathrm{F} 0=$ Force from load cell

$\mathrm{F} 1=$ Upper force value

F2 = Lower Force value

$\mathrm{N} 0=$ Number of nose pokes to end session

N1 = Number of nose pokes

\subsubsection{Pseudocode for the dynamic and static processes}

1. Start Timer

a. If dynamic

i. Get T1, Ro, Ratio value, F1, R1

ii. Start Sampling transducers

1. If $\mathrm{F} 0>=\mathrm{F} 2$,

a. Send data to spreadsheet

b. If nose poke device infrared beam is broken,

i. If ratio value equals $\mathrm{R} 1$

1. Activate dispenser.

2. If head entry device beam is broken,

a. Proceed with session.

3. Else

a. Repeat

ii. Else

1. Wait.

c. Else

i. Wait

2. Else

a. Keep sampling

b. Else

i. Get N0, T3, F1, F2, ratio value

ii. If $\mathrm{F} 0>=\mathrm{F} 2$,

1. Send data to spreadsheet

a. If $\mathrm{F} 0>=\mathrm{F} 1$,

i. Note time $\mathrm{T} 2$

ii. If $\mathrm{T} 2=\mathrm{T} 1 \mathrm{AND} \mathrm{R} 1=$ ratio value 
1. Activate dispenser

2. If head entry device beam is broken

a. Proceed

3. Else

a. Wait

iii. Else

1. Repeat

b. Else

i. Keep sampling

2. Stop

The test procedure is very similar to the conditioning procedure. The nose poke device used in the training procedure is replaced by the custom designed stimulus light fixture described earlier in the section of the nose poke device. The dynamometer is set up as shown in Figure 4 with the transducers in place. The entire process is automated using the LabVIEW software. The user and the system interface is the front panel of the program. The user-friendly front panel makes it fast and easy to set the desired parameters for the data acquisition without further interference on part of the user during the session.

The user initially selects the mode of testing (concentric and isometric or eccentric). Depending on this selection, parameters like time to hold up weights, \# of responses to end session, force threshold, ratio value and the time to end the session are set using the front panel. When the program is executed, a timer is first started and the load cell and the displacement transducer are sampled at a $100 \mathrm{kHz}$.

The operantly conditioned rat enters the Plexiglas tube from the cubicle when the stimulus light is turned on. The yoke is locked at a set height to record the isometric force when the rat performs the lift. The rat enters the tube and steps on the plate and inserts its neck into the ring. Weights may or may not be placed in the weight pans depending on the load required. After inserting its neck into the ring the rat pushes against the yoke assembly and tries to reach up and touch the nose poke device with its snout. The rats are trained to perform a heel raise when inside the tube. When the rat tries to lift the tightly locked yoke assembly, it pushes against the force plate thus developing an isometric force. Since the system has been designed solely to measure the reaction forces developed due to the plantar flexors, when the rat pushes down against the force plate, the forces developed will be due to the plantar flexor muscle. Figure 17 is a flow diagram depicting the flow of data for the dynamic mode of testing.

If the force from the load cell exceeds the lower force value set by the user, then it is sent to the database and all values below this lower force value are discarded. Once the rat begins to push down on the force plate, the forces developed increases. When this value exceeds the upper force value set by the 
user initially, a timer starts recording time. When this time is equals the "time to hold up weights" defined by the user, this is considered a response. When the number of responses is equal to the ratio value, which is also set by the user, a food pellet is rewarded to the rat. The food pellet drops into a food trough and simultaneously the stimulus light and white noise are turned off indicating to the rat to go and get its food. The software is so designed that the session does not proceed until the rat has retrieved the food pellet. Until then, the timer goes on measuring the elapsed time. 


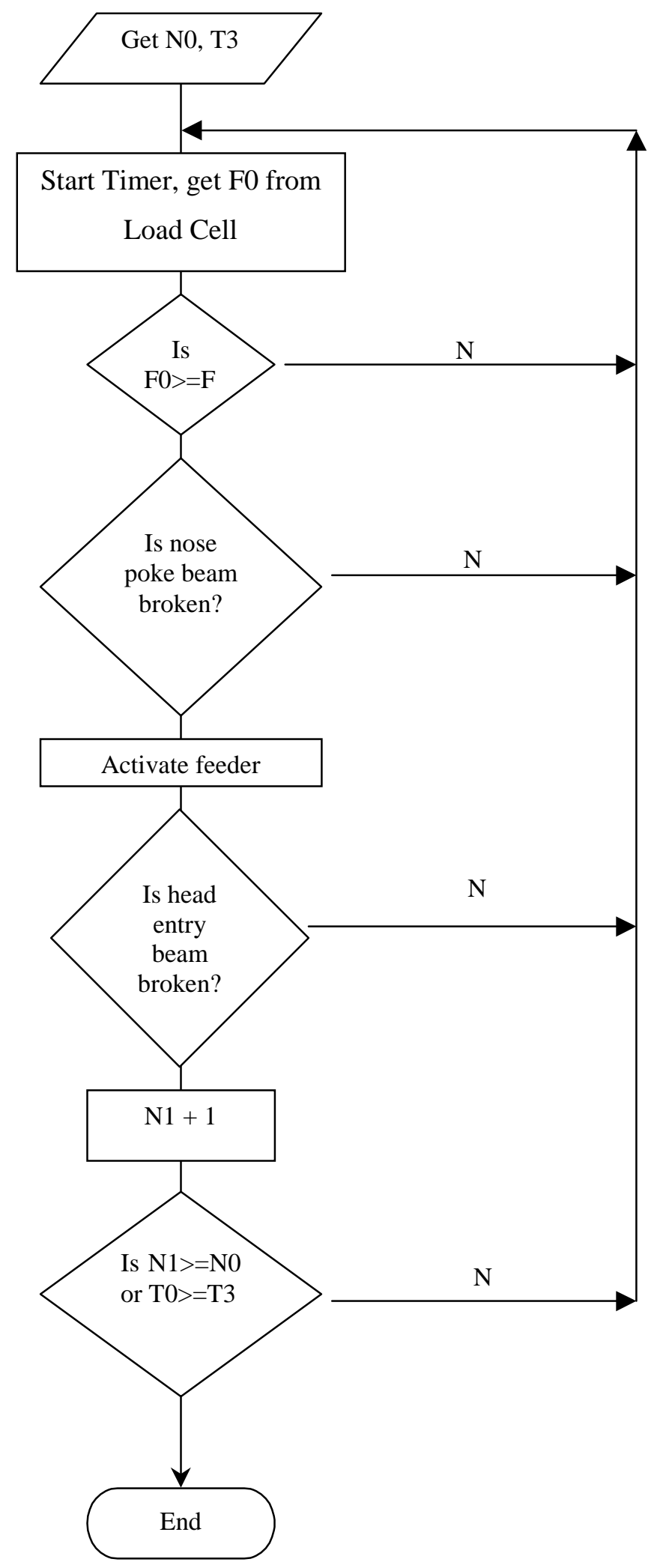

Figure 20-Flowchart for the dynamic process 
As soon as the rat trips the infrared beam of the head entry device while retrieving the food pellet the white noise is offset for a time defined by the user and the stimulus light is turned off until the rat breaks the beam in the trough. The rat goes back in the tube to begin lifting the yoke assembly all over again. The program compares the elapsed time with the time to end session and the number of responses with the number of responses to end the session and when either of the conditions is met, the session is terminated. So, the elapsed time, number of responses and the force values are all stored in a database for further analysis.

In case of the dynamic lift, the user has to define parameters like the number of nose pokes to end the session, time to end the session and also the ratio value. The ratio value determines how many responses are needed for a food pellet reward. The rat performs the task of a "heel raise" after inserting its neck in the ring. In this case, the yoke is not locked at any position. In the dynamic mode, the rat can be tested for during lifting and lowering tasks.

As the program is executed, the timer starts just as in the case of the isometric mode. The force and displacement transducers are sampled at the rate of $100 \mathrm{kHz}$. The white noise, house light and stimulus light are turned on signaling to the rat that the session has started. The rat steps into the Plexiglas tube and onto the force plate. If the force from the load cell is greater than the lower value of force set by the user, it is recorded to the database or discarded otherwise. The rat inserts its neck in the ring and lifts up the yoke and also stands up on its toes. The program then waits for the rat to trip the infrared beam in the nose poke device. When the rat pushes up the yoke assembly all the way up to the nose poke device and sticks its snout into the nose poke device, this is considered a response. When the number of responses equals the ratio value a food pellet is rewarded. Now the program completely stops except for the elapsed time counter until the rat retrieves the food pellet. Eventually, when the rat trips the infrared beam in the food tray the session continues. The program continuously checks for the number of responses and the time set by the user to end the session. The following figure is a flow diagram depicting the flow of data in the isometric mode of testing.

From an electrical standpoint, when the program is executed the LabVIEW program sends out a signal to the load cell and the displacement transducer. The transducers in turn send back voltage signals proportional to the load and displacement. This analog signal that is usually a $+5 \mathrm{~V}$ is sent out to the PCIMIO-16XE-10 A/D board previously described. These analog signals are converted to 12- bit values and 


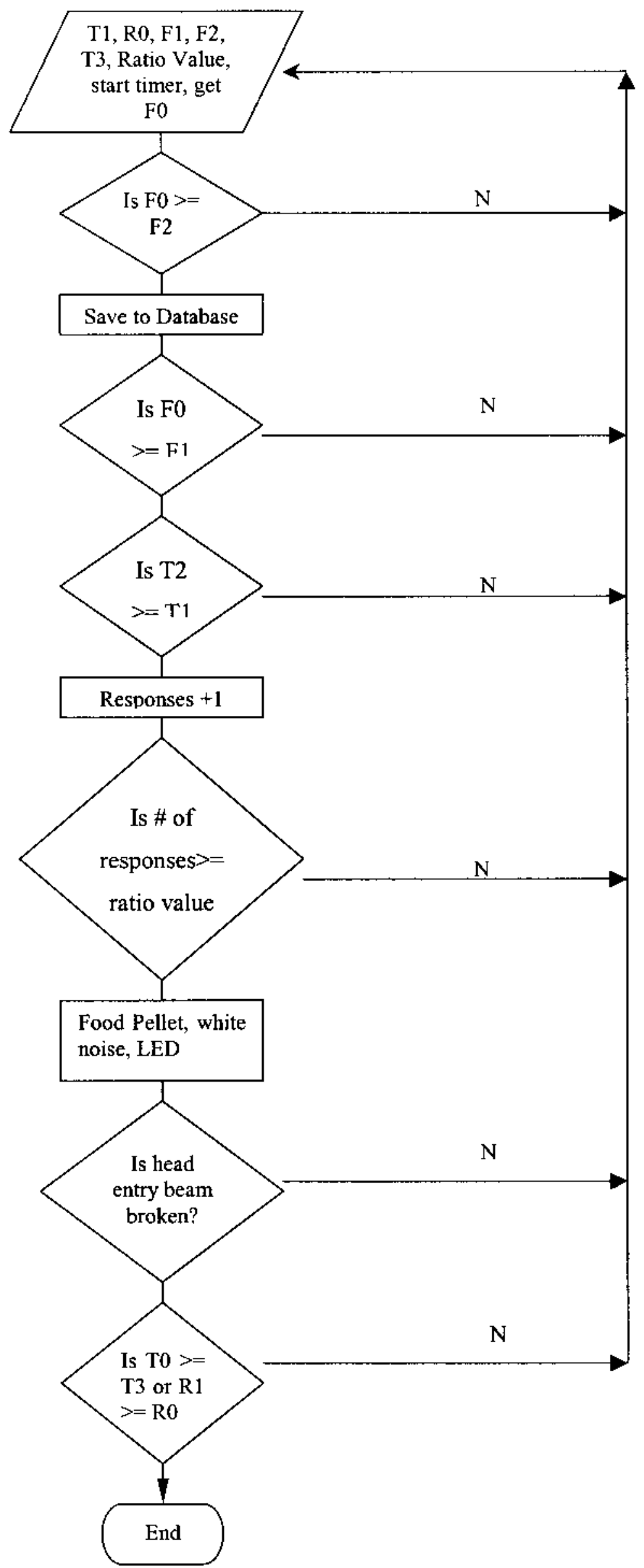

Figure 21- Flowchart for the static process 
sent out to the 2310 signal-conditioning amplifier. The conditioning amplifier amplifies the low level signals and relays it back to the A/D board. The displacement transducer works in the same manner, however, it uses its own custom-built signal conditioner. These amplified signals are either displayed on the monitor in real time or recorded on the database or both. If the conditions are met the software program sends out signals to the PCI- PDISO16 relay card to which are connected all the input and output devices. When the desired responses have been met the program sends out a signal to the relay card that in turn activates the corresponding device. 


\section{CHAPTER 4 \\ CONCLUSIONS}

\subsection{Conclusion}

An extensive literature search resulted in the need for an apparatus that could measure voluntary muscular performances in vivo. The present models do not account for controlled biomechanical exposures. Using noninvasive techniques, the rat can be tested on a dynamometer that can measure time, displacement and force generated by the muscle. With a noninvasive in vivo assessment, multiple exposures are possible, the threshold of injury can be determined easily, and the time course of degeneration and regeneration of muscle fibers can be estimated.

A dynamometer was designed to measure reaction forces and displacement of the lifting response and display it in real time. The entire operation is computer automated completely eliminating human intervention during the session. The operantly conditioned rat enters the Plexiglas tube through an opening and inserts its neck into the ring connected to the yoke. Different weights can be placed in the weight pans to vary the loads. If the rat is being tested in the dynamic mode, the rat lifts up the entire yoke assembly on its shoulders and performs a "heel raise" thus developing force directly in the plantar flexor muscles. Hence, injury can be studied as a cause of the repetitions of the lift or varying loads. The dynamometer is also designed to measure the isometric force developed in the plantar flexor muscles. The data will be analyzed to compare the force developed under different conditions. The dynamometer is equipped with a transducer to measure the range of motion of the muscle from which the velocity and acceleration of the lift can be determined.

The apparatus can be used to study the effects of volitional eccentric, concentric, and isometric muscle actions and varying work-rest cycles on muscle performance, behavior, and histological and biomechanical response. Muscle responses due to controlled biomechanical exposures will enable a thorough understanding of the injury and recovery process.

\subsection{Recommendations}

The apparatus was very effective for measuring the volitional muscular forces in vivo. The dynamometer was capable of displaying the force, range of motion of the plantar flexors in real time as well as enable studies in the dynamic and static modes. This model being a prototype has room for improvements in the design of the setup. The pilot studies conducted on the present setup enabled the reconsideration of a few design factors especially in making it more flexible for the testing of rats of different sizes. 
The dynamometer that might be built after this has been completely tested for functional performance should have the Plexiglas tube 3 inches longer to accommodate large sized rats. The center ring which is of fixed size should be modified to make it more adaptable to allow rats of different size to be tested. The nose poke device, which sits on top of the stimulus light, can be moved out of the way to extend the height of the tube and also a disc should be mounted in place of the aluminum ring on the stimulus light to prevent the rats from chewing on the electrical wires. The position of the pellet dispenser should be rearranged inside the chamber to allow the smooth delivery of the food pellets. The slot machined under the force plate to accommodate the wires form the load cell should be machined so it runs from behind the Plexiglas tube. Finally, to avoid the bearing play, two linear bearing should be used on each shaft to stabilize the movement of the yoke and prevent any unwanted movements. 


\section{REFERENCES}

1. Agarwal, G. L, G. C. Gottlieb. Response to sudden torques about ankle in man: Myotic reflex. J. Neurophysiology 42: $91-96,1979$.

2. Armstrong, R. B, Ogilvie, R. W, Schwane, J. A. Eccentric exercise induced injury to rat skeletal muscle. J Applied Physiology 54: 80 - 93, 1983.

3. Armstrong, R. B. Muscle damage and endurance events. Sports medicine, Volume 3, 1986.

4. Ashton-Miller, J.A, Youda, He, V. A. Kadhiresan, D. A. McCubbery, and J. A. Faulkner. In vivo measurement of mouse ankle properties: An apparatus to measure in vivo biomechanical behavior of dorsi - and plantarflexors of the mouse ankle. Biophysics, Volume 59: 1991.

5. Ayoub, M. M, J. L. Selan, H. C. Chen. Human strength as a predictor of lifting capacity. Proceedings of the human factors society- $30^{\text {th }}$ annual meeting: 960-963, 1986.

6. Caiozzo, V. J, Eugene Ma, S. A. Mc Cue, E. Smith, R. E. Herrick, and K. M. Baldwin. Myosin isoforms and altered activity: A new animal model for modulating myosin isoforms expression by altered mechanical activity. American physiological. Society: 1432 - 1440, 1992.

7. Chaffin, D. B, Herrin, G. D and Keyserling, W. M. Preemployment strength testing, an updated position. Journal of Occupational Medicine 20(6): 403 - 408, 1978.

8. Clarkson, P. M, P. Litchfield, J. Graves, J. Kirwan and W. C. Byrnes. Serum creatine kinase activity following forearm flexion isometric exercise. European Journal of applied. Physiology Occupational Physiology 53: 368 - 371, 1985.

9. Cutlip, R. G, W. T. Stauber, R. H. Willison, T. A. McIntosh and K. H. Means. Dynamometer for rat plantar flexor muscles in vivo. Communication. Medical Biology Engineering Computers, 35: $540-543,1997$.

10. Evans, W. A. The relationship between isometric strength of Cantonese males and the US NIOSH guide for manual lifting. Applied ergonomics: 21.2, 135-142, 1990.

11. Faulkner, J. A and D. A. Jones. An apparatus for unilateral in situ exercise of the lower limb muscles of small mammals. Physiological Society: 11P, March 1985.

12. Faulkner, J. A, S. V. Brooks and J. A. Oplteck. Injury to skeletal muscle fibers during contractions: Conditions of occurrence and prevention. Physical therapy volume 73: 911 - 921, 1993.

13. Friden, J, M. Sjostrom and B. Ekblom: Myofibrillar damage following intense eccentric exercise in man. International Journal of sports Medicine.4: 170 - 176, 1983.

14. Graves, J, P. M. Clarkson, J. Kirwan and P. Litchfield. Serum creatine kinase levels following three different isometric exercise regimes. Medical Science. sports exercise 16: 186 - 187, 1984. 
15. Gregor, R. J, R. R. Roy, W. C. Whiting, R. G. Lovely, J. A. Hodgson and V. R. Edgerton. Mechanical output of the cat soleus during treadmill locomotion: In vivo VS In Situ. J. Biomechanics volume 21, \#9: 721 - 732, 1988.

16. Holbein, M. A and Chaffin B. D. stability limits in extreme postures: Effects of load positioning, foot placement and Strength. Human factors, 39 (3): 456 - 468, 1997.

17. Jones, D. A, D. J. Newham, J. M. Round and S. E. J. Tolfree. Experimental human muscle damage: morphological changes in relation to other indices of damage. Journal of Physiology. London 375: 435 - 448, 1986.

18. Kelsey, J. L and White A. A. Epidemiology and impact of low back pain. Spine 5(2): 133 - 142, 1980.

19. Klitgaard, H. A model for quantitative strength training of hind limb muscles of the rat. Special Comm. The American Physiology Society: 1740 - 1745, 1988.

20. Komulainen, J, Kytola J, Vihko V. Running- induced muscle injury and myocellular enzyme release in rats. J. Applied Physiology 77: 2299- 2304, 1994.

21. Kumar, S. Load history and backache among institutional aids. In Mital A (ed) Advances in Ergonomics 1, Taylor \& Francis, London: 757 - 765, 1989.

22. Kumar, S. Cumulative load as a risk factor for low back pain Spine 15: 1311 - 1316, 1990a.

23. Lieber, R. L, Thornell, L. E and Friden, J. Muscle cytoskeletal disruption occurs within the first 15 min of cyclic eccentric contraction. American Physiological society: 278- 284, 1996.

24. Lowe, D. A., G. L. Warren, C. P. Ingalls, D. B. Boorstein and R. B. Armstrong. Function and protein metabolism of injured muscle: Muscle function and protein metabolism after initiation of eccentric contraction - induced injury. American Physiological society: 1260 - 1270, 1995.

25. Mc Cully, K. K, Faulkner J. A. Injury to skeletal muscle fibers of mice following lengthening contractions. Journal of Applied Physiology. 59: 119- 126, 1985.

26. National Research Council. Work-related musculoskeletal disorders: A review of the evidence. 1998

27. Newham, D. J, D. A. Jones, S. E. J. Tolfree and R. H. T. Edwards. Ultra structural changes after concentric and eccentric contractions. Journal Neural Science 61: 109-1122, 1983.

28. Newham, D. J, D. A. Jones and R. H. T. Edwards. Large delayed plasma creatine kinase changes after stepping exercise. Muscle nerve 6: 380 - 385, 1983.

29. Newham, D. J, D. A. Jones and R. H. T. Edwards. Plasma creatine kinase changes after eccentric and concentric contractions. Muscle nerve 9: 59-63, 1986.

30. NIOSH. Work practices guide for manual lifting. Department of Health and Human Services, Cincinnati, USA: 81- 122, 1981. 
31. Patel, T. J, D. Cuzion, O. Mathieu-Costello, J. Frieden and R. L. Lieber. Increased oxidative capacity does not affect muscle injury: Increased oxidative capacity does not protect skeletal muscle fibers from eccentric contraction - induced injury. American physiology Society: R 1300 - R 1308, 1998.

32. Pitman, M. I and Peterson. L. Biomechanics of skeletal muscle. Chapter 5: 89-107, 1990.

33. Sola, O. M., D. L. Christensen, and A. W. Martin. Hypertrophy and hyperplasia of adult chicken anterior latissimus dorsi muscles following stretch with and without denervation. Exp. Neurology, 41: 76-100, 1973.

34. Stauber, W. T. Exercise and sport sciences reviews: Eccentric action of muscles: Physiology, injury and adaptation. Volume 17: 158-185, 1989.

35. Warren, G. L, D. A. Lowe, C. L. Inman, O. M. Orr, H. A. Hogan, S. A. Bloomfield and R. B. Armstrong. Functional Matching of muscle and bone: Estradiol effect on anterior crural muscles - tibial bone relationship and susceptibility to injury. American physiological society: 1660 $1665,1996$.

36. Warren, G. L, D. A. Lowe and R. B. Armstrong. Measurements of functional muscle injury: Measurement tools used in the study of eccentric contraction - induced injury. Sports Med. 27: $44-59,1999$.

37. Willems, M. E. T and W. T. Stauber. Performance of skeletal muscle in intact rat: Isometric and concentric performance of electrically stimulated ankle plantar flexor muscles in intact rat. Exp. Physiology 84: 379 - 3891999.

38. Zajac, F. E. Muscle and tendon: Properties, models, scaling, and application to biomechanics and motor control. Volume 17, Issue 4: 359- 411, 1989.

39. LabVIEW- User Manual, Version 5.1, National Instruments, January 2000.

40. LabVIEW- Reference Manual, Version 5.1, National Instruments, January 2000.

41. LabVIEW Programming, Data Acquisition and Analysis- Jefferey y. Beyon, 2001.

42. U. S. Department of Labor. Occupational injuries and illnesses in the United States by industry. Bureau of Labor Statistics Bulletin 23399, U.S Department of Labor, Washington, D.C, 1996. 


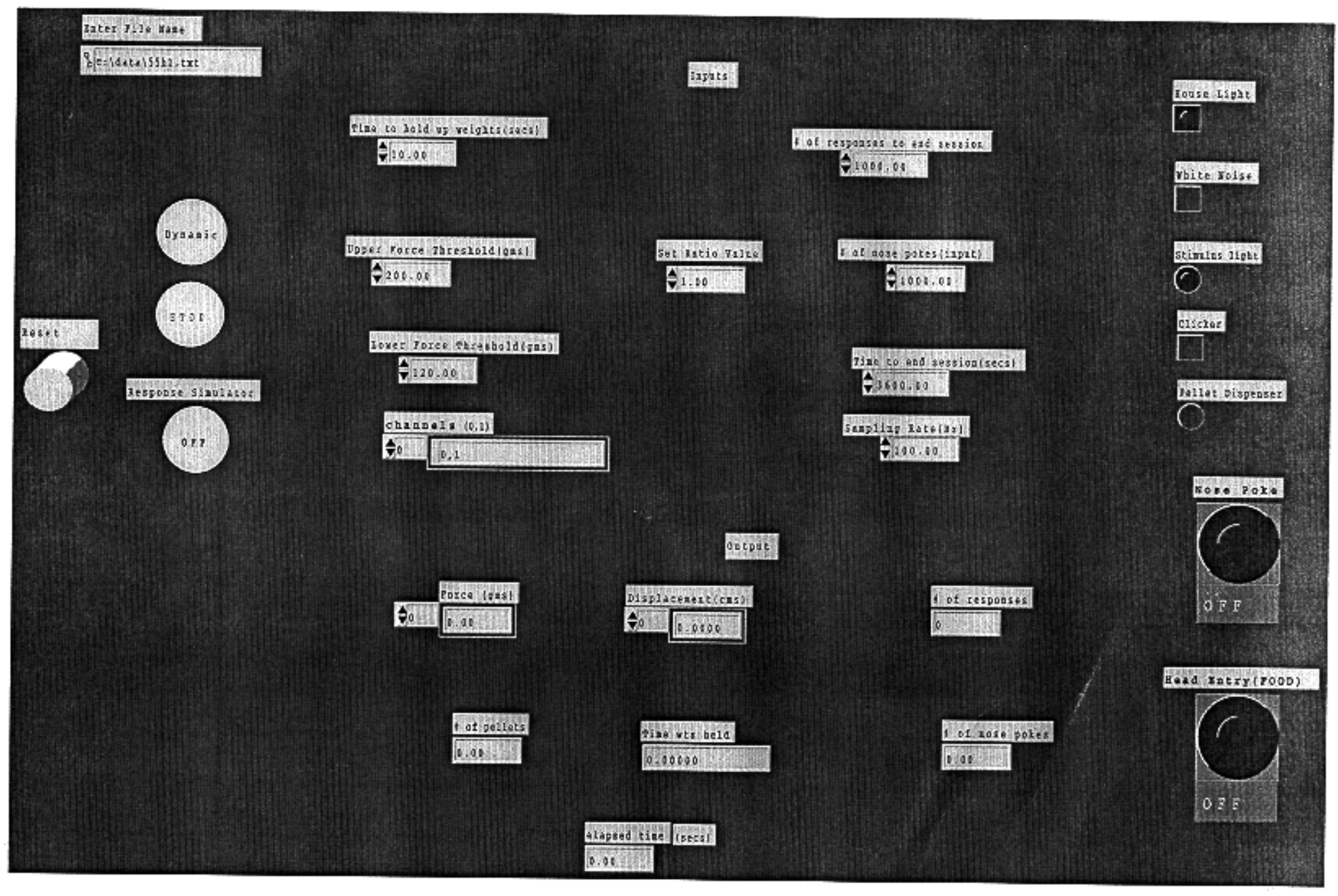

Figure A1: Front Panel 


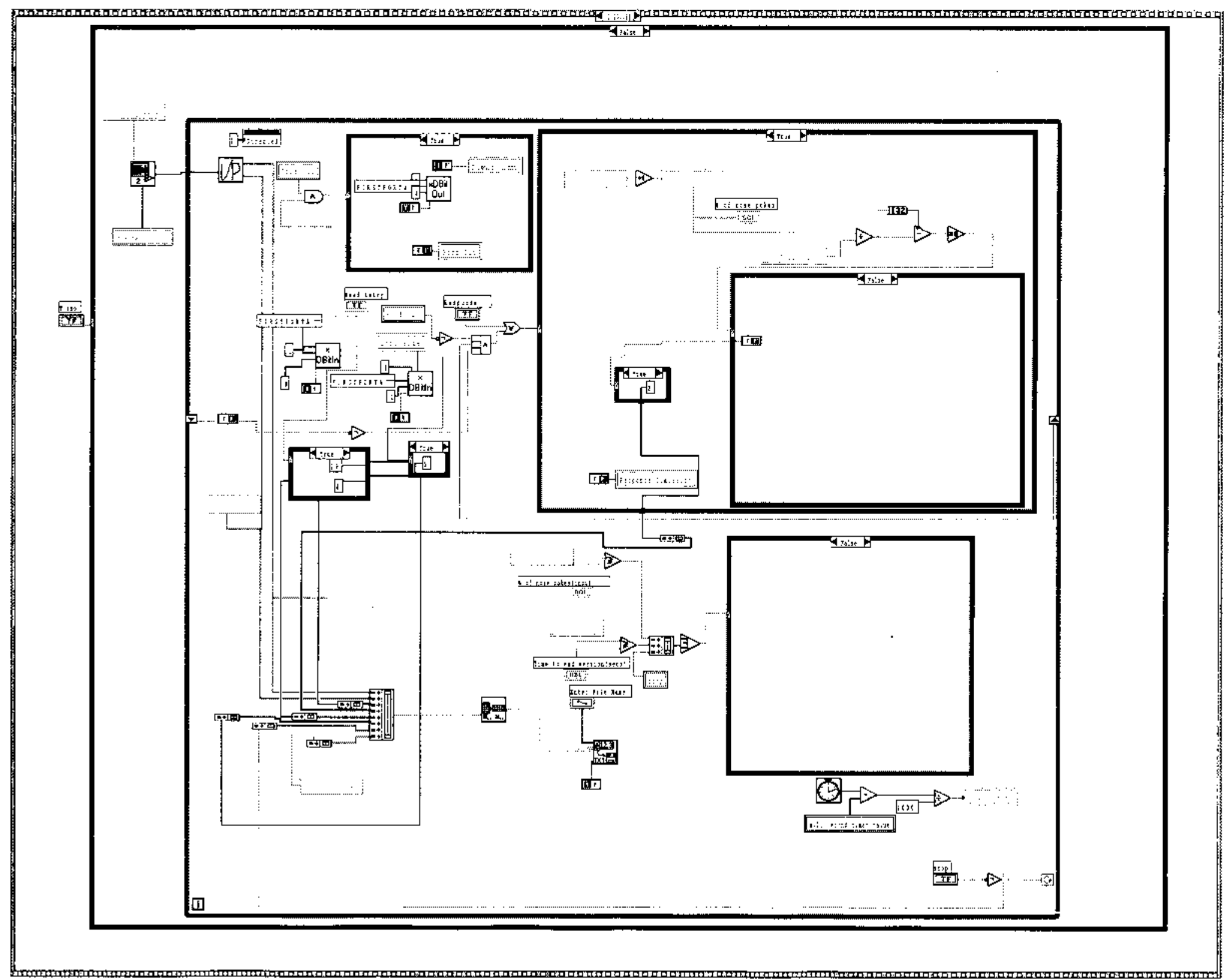

Figure A2: Dynamic Process 

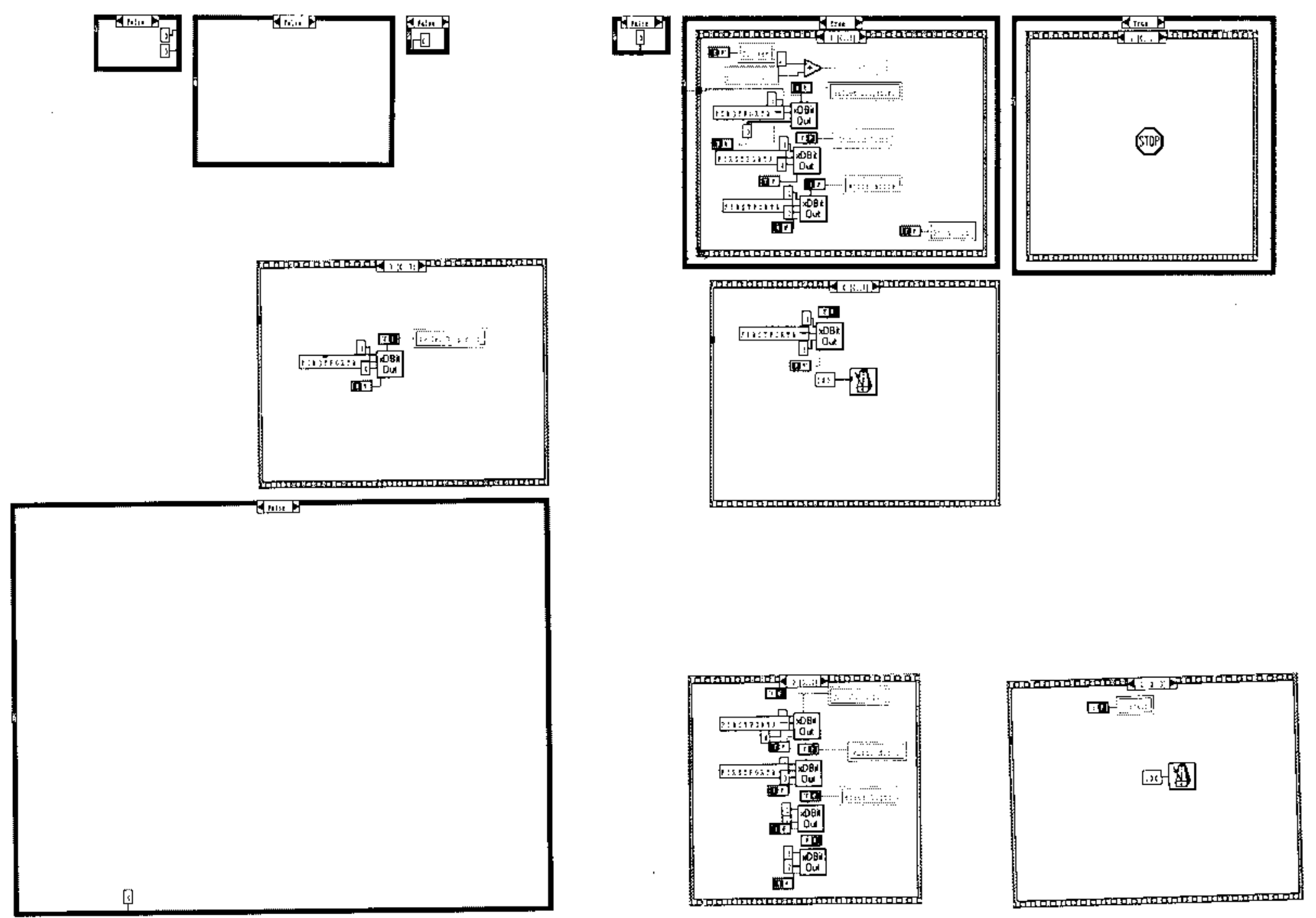

Figure A3: Dynamic Process (Contd.) 


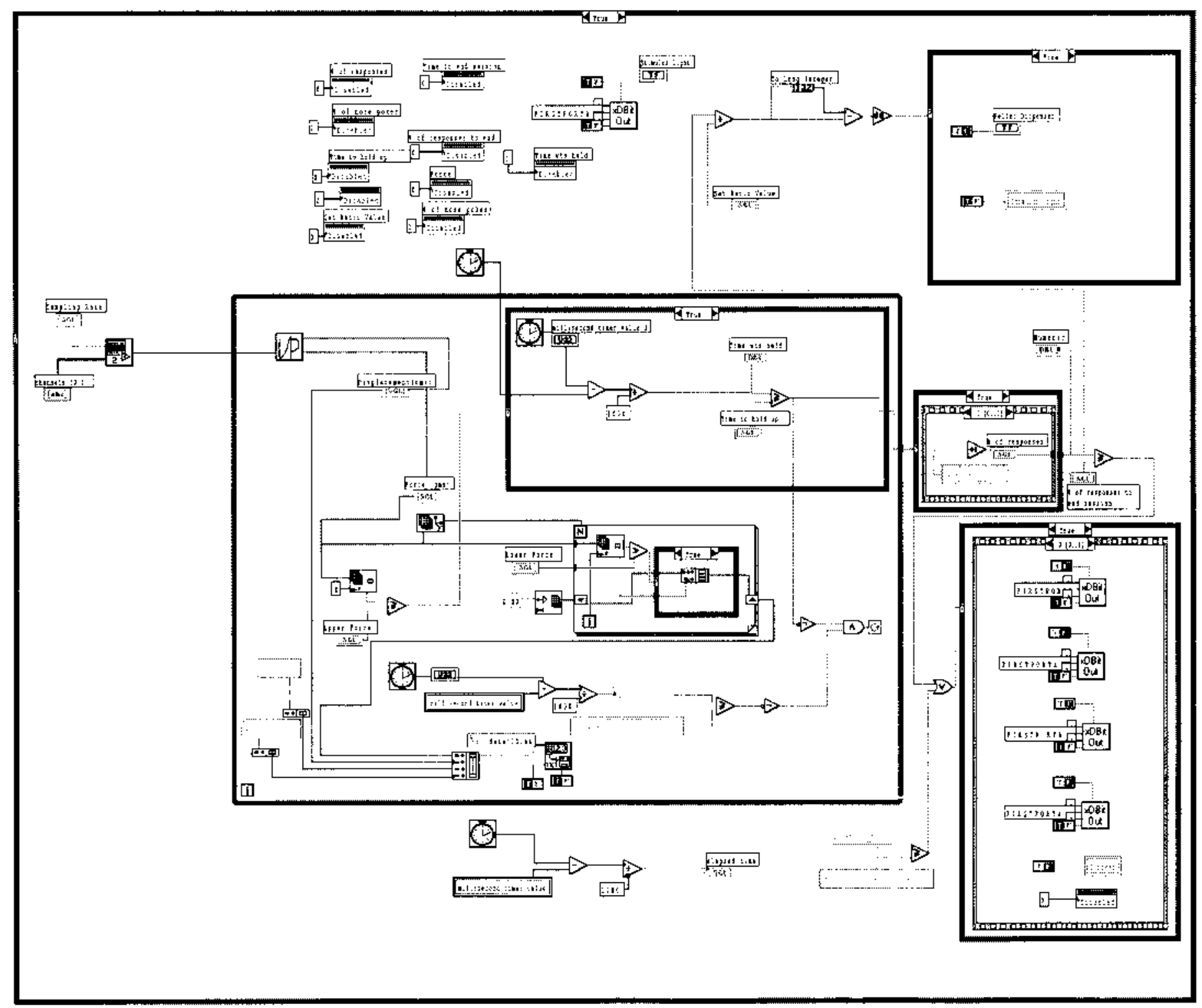

Figure A4: Static Process 


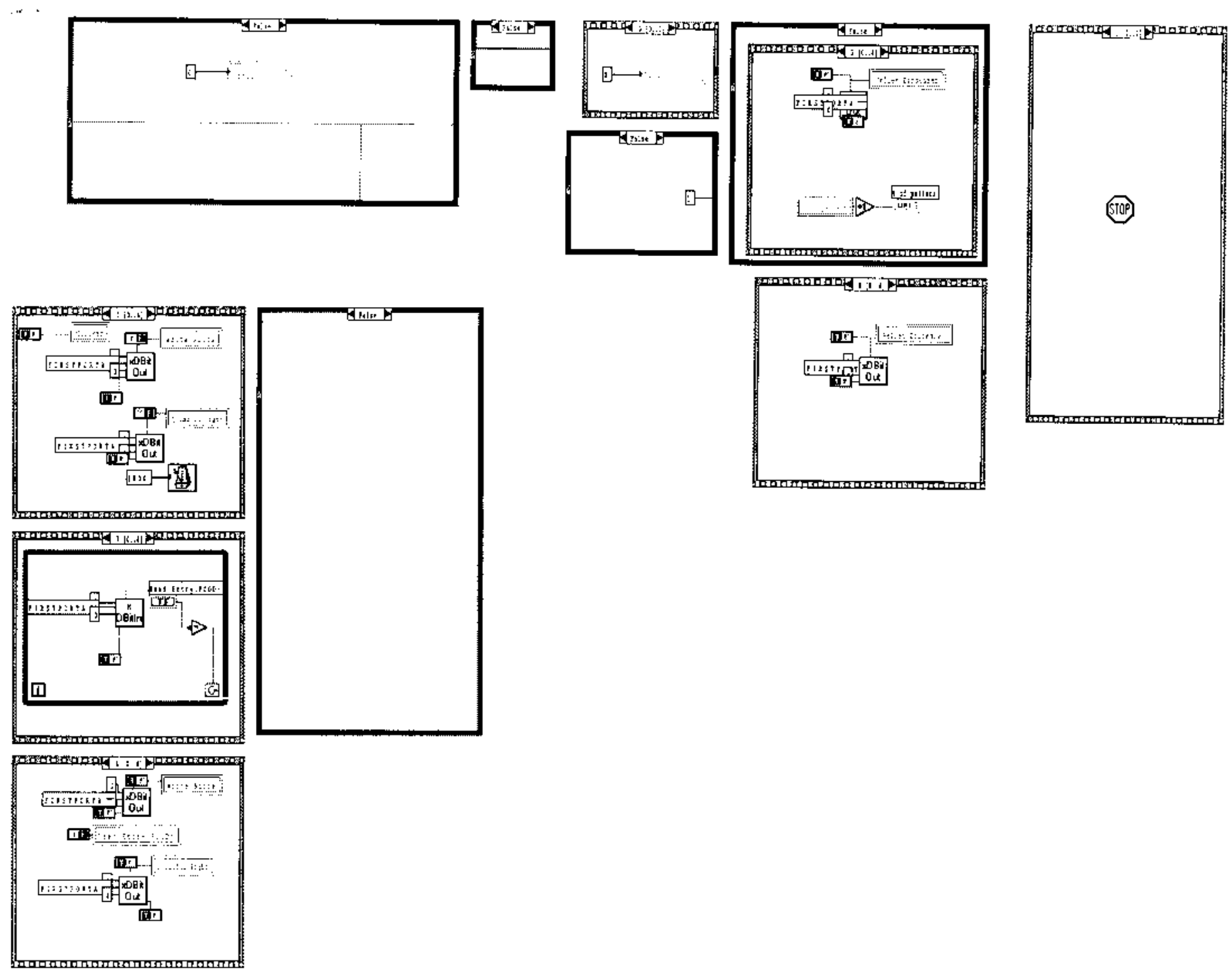

Figure A5: Static Process (Contd.) 


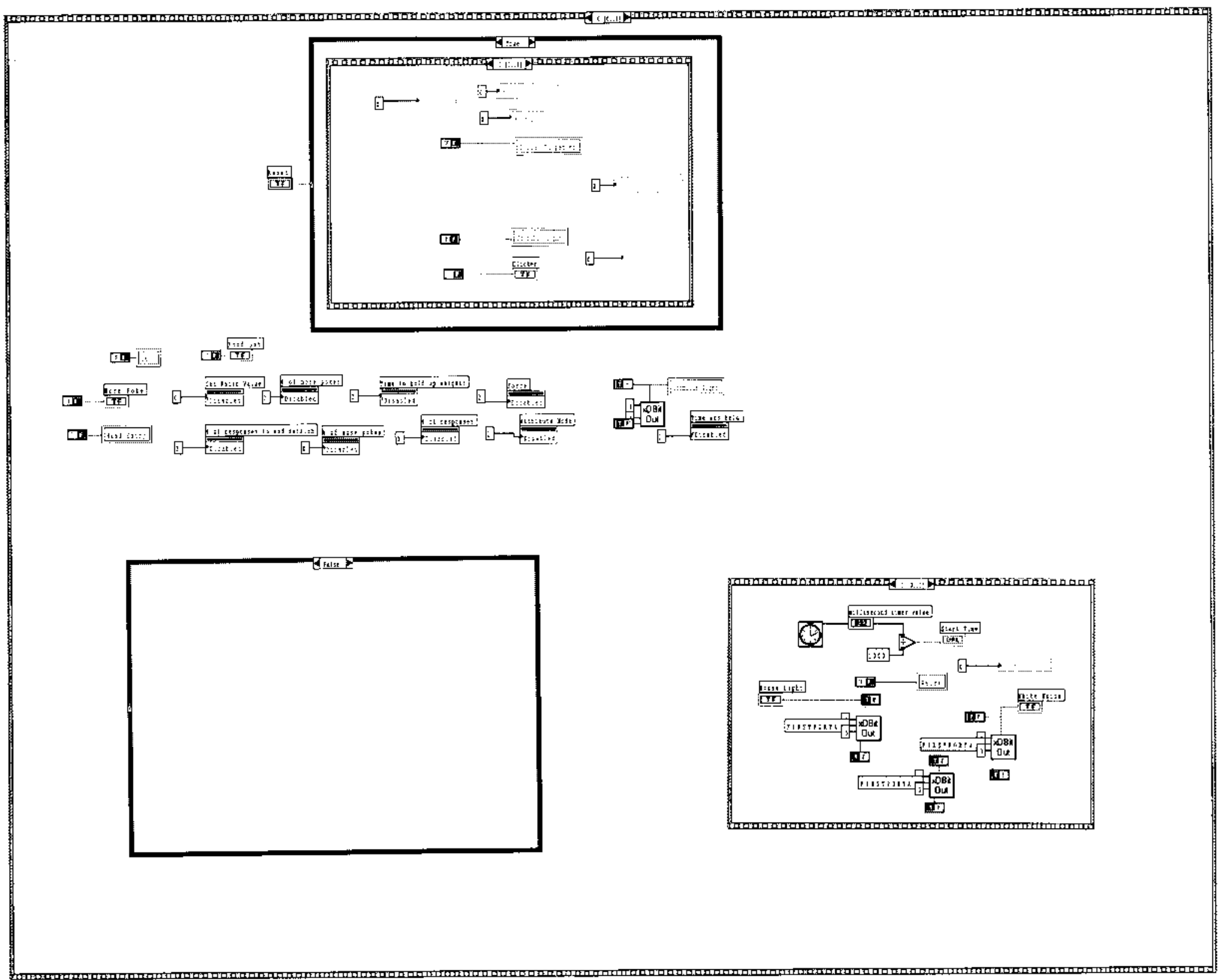

Figure A6: Reset Process 